\title{
The mysterious morphology of MRC0943-242 as revealed by ALMA and MUSE
}

\author{
Bitten Gullberg ${ }^{1}$, Carlos De Breuck ${ }^{1}$, Matthew D. Lehnert ${ }^{2}$, Joël Vernet ${ }^{1}$, Roland Bacon ${ }^{3}$, Guillaume Drouart ${ }^{4}$, \\ Bjorn Emonts ${ }^{5}$, Audrey Galametz ${ }^{6}$, Rob Ivison ${ }^{1,7}$, Nicole P. H. Nesvadba ${ }^{8}$, Johan Richard ${ }^{3}$, Nick Seymour ${ }^{9}$, \\ Daniel Stern ${ }^{10}$, and Dominika Wylezalek ${ }^{11}$ \\ ${ }^{1}$ European Southern Observatory, Karl-Schwarzschild-Str. 2, 85748 Garching, Germany \\ e-mail: bgullber@eso.org \\ 2 Institut d'Astrophysique de Paris, UMR 7095, CNRS, Université Pierre et Marie Curie, 98bis boulevard Arago, 75014 Paris, France \\ 3 CRAL, Observatoire de Lyon, CNRS, Université Lyon 1, 9 avenue Ch. André, 69561 Saint Genis-Laval Cedex, France \\ 4 Department of Earth and Space Science, Chalmers University of Technology, Onsala Space Observatory, 43992 Onsala, Sweden \\ 5 Centro de Astrobiología (INTA-CSIC), Ctra de Torrejón a Ajalvir, km 4, 28850 Torrejń de Ardoz, Madrid, Spain \\ ${ }^{6}$ Max-Planck-Institut für Extraterrestrische Physik, Giessenbachstraße 1, 85748 Garching, Germany \\ 7 Institute for Astronomy, The University of Edinburgh, Royal Observatory, Blackford Hill, Edinburgh EH9 3HJ, UK \\ ${ }^{8}$ Institut d'Astrophysique Spatiale, CNRS, Université Paris-Sud, Bat. 120-121, 91405 Orsay, France \\ 9 International Centre for Radio Astronomy Research, Curtin University, Perth WA 6845, Australia \\ 10 Jet Propulsion Laboratory, California Institute of Technology, Pasadena, CA 91109, USA \\ 11 Department of Physics and Astronomy, Johns Hopkins University, 3400 N. Charles St, Baltimore, MD 21218, USA
}

Received 30 June 2015 / Accepted 9 October 2015

\section{ABSTRACT}

\begin{abstract}
We present a pilot study of the $z=2.923$ radio galaxy MRC0943-242, where we combine information from ALMA and MUSE data cubes for the first time. Even with modest integration times, we disentangle the AGN and starburst dominated components. These data reveal a highly complex morphology as the AGN, starburst, and molecular gas components show up as widely separated sources in dust continuum, optical continuum, and $\mathrm{CO}$ line emission observations. $\mathrm{CO}(1-0)$ and $\mathrm{CO}(8-7)$ line emission suggest that there is a molecular gas reservoir offset from both the dust and the optical continuum that is located $\sim 90 \mathrm{kpc}$ from the AGN. The UV line emission has a complex structure in emission and absorption. The line emission is mostly due to a large scale ionisation cone energised by the AGN, and a Ly $\alpha$ emitting bridge of gas between the radio galaxy and a heavily star-forming set of components. Strangely, the ionisation cone has no $\operatorname{Ly} \alpha$ emission. We find this is due to an optically thick layer of neutral gas with unity covering fraction spread out over a region of at least $\sim 100 \mathrm{kpc}$ from the AGN. Other less thick absorption components are associated with Ly $\alpha$ emitting gas within a few tens of kpc from the radio galaxy and are connected by a bridge of emission. We speculate that this linear structure of dust, Ly $\alpha$ and CO emission, and the redshifted absorption seen in the circum nuclear region may represent an accretion flow feeding gas into this massive AGN host galaxy.
\end{abstract}

Key words. galaxies: evolution - galaxies: high-redshift - galaxies: active - galaxies: ISM - galaxies: halos

\section{Introduction}

Powerful high- $z$ radio galaxies $(\mathrm{H} z \mathrm{RGs})$, defined as having $L_{3 \mathrm{GHz}}>10^{26} \mathrm{~W} \mathrm{~Hz}^{-1}$ and $z>1$, are unique markers of the most active galaxies in the early Universe, showing signatures of both luminous AGN activity and vigorous starbursts. $\mathrm{HzRGs}$ are extremely luminous in both the mid-IR (Ogle et al. 2006; Seymour et al. 2007; De Breuck et al. 2010) and the submillimetre (submm) waveband (Archibald et al. 2001; Reuland et al. 2004; Drouart et al. 2014). This has been interpreted as evidence of high black hole accretion rates, combined with high star formation rates (SFRs). $\mathrm{H} z \mathrm{RG}$ are some of the most massive galaxies known at any redshift, with $M>10^{11} M_{\odot}$ of stars (Seymour et al. 2007; De Breuck et al. 2010), confirming prior indications of their large masses from the tight correlation of the observed near-IR Hubble $K-z$ diagram for these sources (Lilly \& Longair 1984; Eales et al. 1997; De Breuck et al. 2002). In order to be so luminous, the black holes in these galaxies must be massive. Given that stellar bulge mass correlates with black hole mass (e.g. Tremaine et al. 2002), it is therefore no surprise that the most powerful radio galaxies reside in the most massive stellar hosts.

Disentangling the AGN and starburst components requires excellent sampling of the spectral energy distribution (SED). Seymour et al. (2012) illustrate the decomposition of the starburst and AGN components using $3.5-850 \mu \mathrm{m}$ photometry in the $z=2.16$ radio galaxy, PKS 1138-262, showing that both have roughly equal contributions to the IR luminosity. Disentangling the SED of $\mathrm{H} z \mathrm{RG}$ is the main goal of our HErschel Radio Galaxy Evolution (HERGÉ) project (Drouart et al. 2014). The 71 HzRG targeted in the HERGÉ project are uniformly distributed across $1.0<z<5.2$ with a range of radio powers. Spitzer, Herschel, SCUBA, and LABOCA data suggest that the sources targeted in the HERGÉ project have very high far-IR (FIR) luminosities $\left(\sim 10^{13} L_{\odot}\right.$, Drouart et al. 2014), but the data do not have high enough spatial resolution to pinpoint the AGN host galaxies as the source of the FIR emission. Resolution is important, as spectral decomposition alone is probably insufficient to understand the evolutionary state of $\mathrm{H} z \mathrm{RGs}$. 
Previous single sources studies of $\mathrm{H}_{z} \mathrm{RG}$ s have revealed that these systems have highly complicated morphologies, for example with line emission separated from the continuum emission. In several systems (e.g. 4C41.17, 4C60.07, TXS0828+193, and B3 J2330+3927), most of the gas and dust emission originates from the companion rather than the AGN host galaxy, while others (e.g. MRC0114-211, MRC0156-252, and MRC2048-272) show no evidence for a companion (De Breuck et al. 2003, 2005; Ivison et al. 2008, 2012; Nesvadba et al. 2009; Emonts et al. 2014). These observations confirm that spectral decomposition alone is insufficient to understand the evolutionary state of $\mathrm{HzRGs}$; sufficiently high spatial resolution to separate out individual emission components is also crucial.

The sub-arcsecond spatial resolution of ALMA will undoubtedly reveal a much more complex composition of AGN and starburst dominated sources than previously thought. To illustrate this point, we conducted a pilot study of MRC0943-242 at $z=2.9$, where we combine - for the first time - sensitive ALMA $235 \mathrm{GHz}$ and VLT/MUSE observations. This combination allows us to trace the spatially resolved distributions of the ionisation state of the warm ionised medium $\left(T \sim 10^{4} \mathrm{~K}\right)$ through the UV emission and absorption lines (e.g. CIV), the warm neutral gas $\left(T \sim\right.$ few $\left.\times 10^{3} \mathrm{~K}\right)$ through Ly $\alpha$ absorption, and the dense and diffuse molecular gas as probed by the dust and high- $J$ CO transitions. Through this combination it is possible to get a comprehensive picture of several important phases of gas in the interstellar medium and haloes of $\mathrm{HzRGs}$.

MRC0943-242 is an ultra-steep spectrum radio source with a spectral index of $\alpha=-1.5$ between $1.4-30 \mathrm{GHz}$ and shows no evidence of a spectral curvature within this range (Carilli et al. 1997; Emonts et al. 2011). MRC0943-242 is located in a proto-cluster (with an over density of almost $5 \sigma$; Wylezalek et al. 2013) surrounded by many nearby companions detected in Ly $\alpha$ with known redshifts (Venemans et al. 2007) and a giant quiescent Ly $\alpha$ halo with a diameter of $\geq 100 \mathrm{kpc}-$ extending far beyond the radio structure (Villar-Martín et al. 2003). A deep absorption trough in the Ly $\alpha$ emission line suggests the presence of a large amount of neutral gas (Röttgering et al. 1995). Based on a $1.5 \AA$ A-resolution spectrum Röttgering et al. (1995) conclude that the saturated absorption trough is due to a reservoir of neutral hydrogen (HI) situated within the $\mathrm{H} z \mathrm{RG}$ it self. They determine a HI column density of $N_{\mathrm{HI}}=10^{19} \mathrm{~cm}^{-2}$ in the absorber. Using the same observational set-up, Binette et al. (2000) determine a HI column density similar to that of Röttgering et al. (1995) and a CIV column density of $N_{\text {CIV }}=10^{14.5 \pm 0.1} \mathrm{~cm}^{-2}$. However based on Voigt-profile fittings to the absorption troughs in both the Ly $\alpha$ and CIV emission lines Binette et al. (2000) conclude that the reservoir of gas causing the absorption features is not situated within the $\mathrm{H}_{z} \mathrm{RG}$, but rather due to a low metallicity $\left(Z=0.01 Z_{\odot}\right)$, low density gas in the outer halo at a redshift of $z=2.29202 \pm 0.0002$. They propose that this gas is a remnant of gas expelled from the $\mathrm{H} z \mathrm{RG}$ during the initial starburst. Though such a starburst is expected to enrich the expelled gas more than what is observed for MRC0943-242, Jarvis et al. (2003) still favoured this scenario. Based on numerical simulations by Bekki (1998), which predict that the outer regions of galactic halos should be less metal-rich, Jarvis et al. (2003), as Binette et al. (2000), favored a picture where the shell lies in the halo to explain its the low metallicity. Furthermore, by comparing MRC0943-242 with another HzRG (MRC0200+015), they argue that the age of the system can have an influence on the metallicity of the shells. They conclude that the metallicities of absorption shells surrounding $\mathrm{H}_{z} \mathrm{RG}$ vary from galaxy to galaxy and from shell to shell. This implies that the shells must be enriched by a variety of processes. With UVES data with a spectral resolution of 25000-40000 Jarvis et al. (2003) identified three additional weaker absorbing components of different HI column densities.

In this paper, we present a study of MRC0943-242, where we for the first time combine relatively short ALMA submm observations with MUSE optical observations. This ALMAMUSE-pilot study reveals an even more complex morphology of MRC0943-242 than previously seen with star-formation taking place far outside the AGN host galaxy. In Sect. 2 we present our ALMA submm and MUSE optical observations. The results of these observations are given in Sect. 3 and are analysed and discussed in Sect. 4. In Sect. 5 we speculate as to the origin and role of the substructures and in Sect. 6 we summarise our conclusion.

\section{Observations}

\subsection{ALMA observations}

The Atacama Large Milimeter/submilimeter Array (ALMA) cycle 1 observations in Band 6 were carried out on 2014 April 29 for $3 \mathrm{~min}$ on-source time with 36 working antennas. The four $1.875 \mathrm{GHz}$ spectral windows were tuned to cover the frequency ranges $233.6-237.1 \mathrm{GHz}$ and $248.6-252.3 \mathrm{GHz}$. We used the Common Astronomy Software Applications (CASA) and the supplied calibration script to calibrate the data, produce the data cube and moment 0 maps. The average $T_{\text {sys }}$ was $\sim 80 \mathrm{~K}$ and the average precipitable water vapour $1.3 \mathrm{~mm}$. The quasar J10372934 was used as bandpass calibrator and results in an astrometric accuracy of 4 mas. The UV coverage was sparse, but uniform over $\sim 400 \mathrm{k} \lambda$. In order to search for $\mathrm{CO}(8-7)$ line emission at $v_{\text {rest }}=921.80 \mathrm{GHz}$, which is within the frequency range at $z=2.923$, we subtracted the continuum in the UV-plane by fitting a first-order polynomial to all four spectral windows, as no strong line emission is contaminating the continuum. As our data are limited by the signal-to-noise ratio $(\mathrm{S} / \mathrm{N})$, we use a natural weighting (robust parameter of 2). This results in a $0{ }^{\prime} 7 \times 00^{\prime} 6$ beam with pa $=75^{\circ}$ and an rms of $0.1 \mathrm{mJy}$ for the continuum. We binned the line data to $10 \mathrm{~km} \mathrm{~s}^{-1}$ which has an rms of $1.7 \mathrm{mJy}$. As objects are detected up to $12^{\prime \prime}$ from the phase centre, we applied a primary beam correction on all our maps and spectra.

\subsection{MUSE observations}

Multi Unit Spectroscopic Explorer (MUSE; Bacon et al. 2010) observations were obtained on 2014 February 21, during the first commissioning run of the instrument (see Bacon et al. 2014) on VLT/UT4. This one hour observation taken under $\sim 1^{\prime \prime}$ seeing was split into three 20 min exposures taken at 45 , 135 and $225 \mathrm{deg}$ position angle with a small dithering offset to minimise the effect of systematics. In order to observe the Ly $\alpha$ line of MRC0943-242 at $476.5 \mathrm{~nm}$, we used the extended wavelength range mode (i.e. without second order blocking filter), resulting in a observed wavelength range of 460-935 nm). These data were taken specifically to compare MUSE observations with previous observations with other instruments of this well-studied source. Thus it serves as a pilot study for using MUSE to study $\mathrm{H}_{z}$ RGs. All data were processed with the version 1.0 of the MUSE pipeline (Weilbacher et al. 2012) to produce a fully calibrated and sky subtracted data cube. Finally, the cube was cleaned for sky subtraction residuals with the principal component analysis (PCA) based algorithm ZAP developed by Soto et al. (in prep.). To analyse the line emission and to remove 

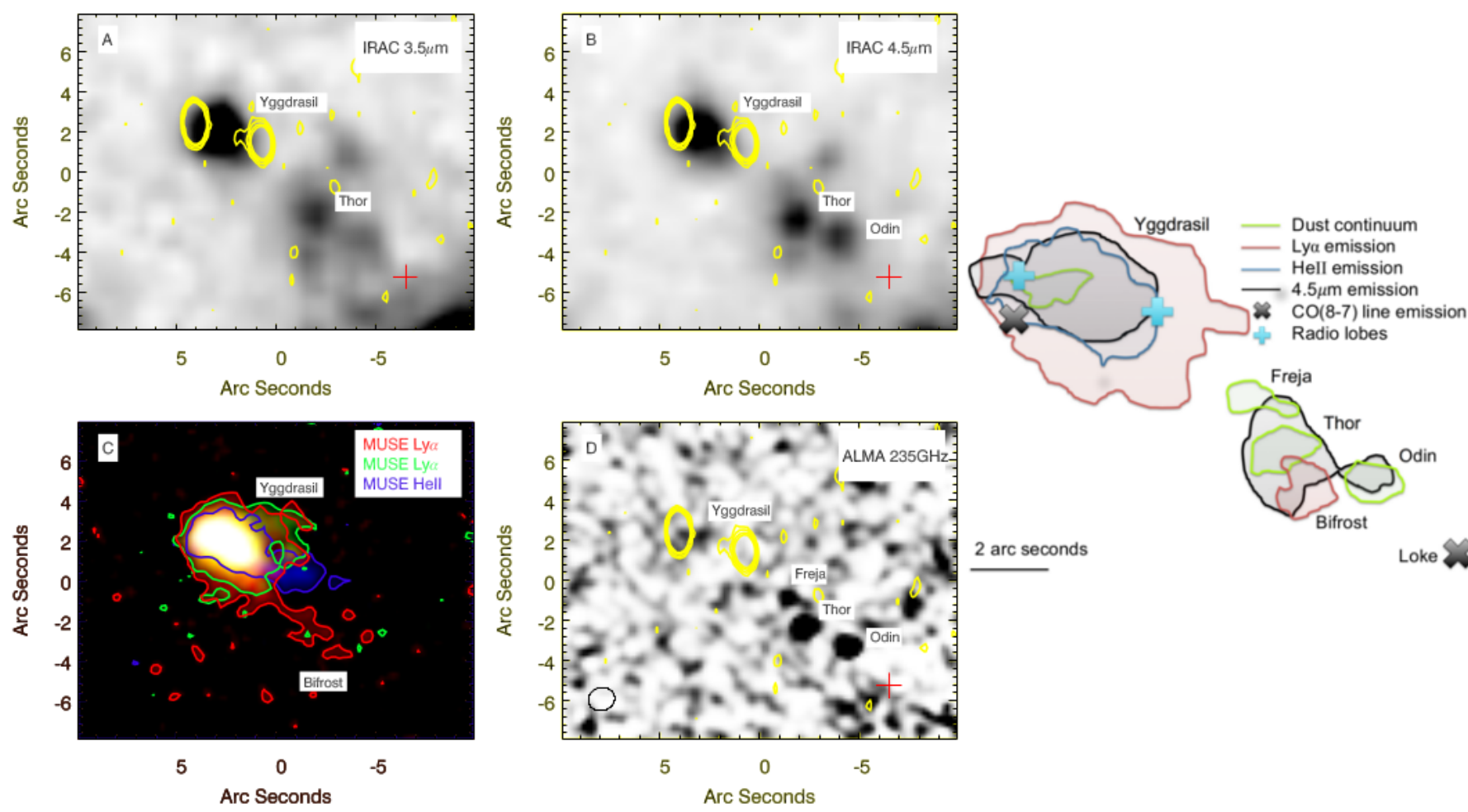

Fig. 1. Overview of the IRAC (3.6 $\mu \mathrm{m}$ and $4.5 \mu \mathrm{m})$, MUSE (Ly $\alpha$ and HeII) and ALMA (235 GHz) maps. Panel A) and B): the IRAC $3.6 \mu \mathrm{m}$ and $4.5 \mu \mathrm{m}$ images showing both Yggdrasil and Thor. Both IRAC images have a spatial sampling of 0.61"/pixel. Panel C): a red (Ly $\alpha)$, green (Ly $\alpha$ ) and blue (HeII) image composed of moment- 0 maps of the MUSE cube, which have an spatial sampling of $0.2^{\prime \prime} /$ pixel. The red Ly $\alpha$ moment- 0 is summed over $\lambda_{\mathrm{obs}}=4768.8-4776.2 \AA$ (see red bar in panel A of Fig. 5), the green Ly $\alpha$ moment -0 is summed over $\lambda_{\mathrm{obs}}=4754.3-4758.8 \AA$ (see green bar in panel A of Fig. 5) and the blue HeII moment-0 map is summed over $\lambda_{\text {obs }}=6422.5-6430.0 \AA$. The red Ly $\alpha$ reveals a bridge of emission connecting Yggdrasil and Bifrost (see Sect. 3), while the green Ly $\alpha$ emission shows extended emission to the west. The Blue HeII shows an extended tail of HeII emission towards the WSW, which is not seen in Ly $\alpha$ emission (see Sect. 3.2). Panel D): the ALMA dust continuum map reveals weak dust emission at the position of the AGN, but strong dust emission in three aligned components 48-65 kpc SW of the AGN. The mm continuum flux density of all four continuum sources are extracted with apertures of; Yggdrasil: $2.6^{\prime \prime} \times 1.7^{\prime \prime}$, Freja: $1.8^{\prime \prime} \times 1^{\prime \prime}$, Thor; $1.9^{\prime \prime} \times 1.5^{\prime \prime}$ and Odin: $1.2^{\prime \prime} \times 1.4^{\prime \prime}$. Far right: schematic overview of the multi-wavelength components detected in MRC0943-242. VLA 4.5 GHz radio observations have been overlaid in yellow contours in Panel A), B), and D). The plotted contour levels are for $-3 \sigma, 2 \times 3 \sigma, 3 \sqrt{2} \times 3 \sigma$, $5 \sqrt{2} \times 3 \sigma$, which is the same for all VLA contour levels through out the paper. The position of Loke is marked with a red cross in Panel A), B), and $\mathbf{D})$.

a few artefacts in the data, we continuum subtracted the cubes around specific emission lines such as $\operatorname{Ly} \alpha$.

\subsection{Previous supporting Spitzer observations}

MRC0943-242 was observed among 68 other $\mathrm{HzRG}$ in the redshift range $1<z<5.2$ in a large Spitzer survey (Seymour et al. 2007). MRC0943-242 was observed with the Infrared Array Camera (IRAC), the Infrared Spectrograph (IRS), and the Multiband Imaging Photometer (MIPS) on Spitzer, which cover the wavelengths $3.6 \mu \mathrm{m}, 4.5 \mu \mathrm{m}, 5.8 \mu \mathrm{m}, 8.0 \mu \mathrm{m}, 16 \mu \mathrm{m}$, $24 \mu \mathrm{m}, 70 \mu \mathrm{m}$ and $160 \mu \mathrm{m}$ (see Seymour et al. 2007 for details on the observations and reductions). At 3.6 and $4.5 \mu \mathrm{m}$, we use the deeper IRAC observations (Fig. 1) obtained as part of the Clusters Around Radio Loud AGN project (CARLA; Wylezalek et al. 2013). The IRAC and MIPS observations reveal that the continuum emission associated with the dust features in our ALMA data is very diffuse and faint (see Sect. 3.3 for more details). In support of the Spitzer data, we also examined the observations of MRC0943-242 from Keck and HST/NICMOS (van Breugel et al. 1998; Pentericci et al. 2001). We do not see any evidence at sub-arcsecond resolution of multiple components.

\section{Results}

Combining for the first time ALMA submm observations with MUSE optical observations reveals an even more complex morphology than seen previously: with multiple components where only the AGN dominated component is visible in all wavelengths. Figure 1 shows the IRAC $3.6 \mu \mathrm{m}$ and $4.5 \mu \mathrm{m}$ image, Ly $\alpha$ and HeII moment- 0 maps in a colour image observed with MUSE and the $235 \mathrm{GHz}$ map observed with ALMA. The Ly $\alpha$ moment- 0 map shows a bright source at the position of the $\mathrm{H}_{z} \mathrm{RG}$ (hereafter Yggdrasil) and an additional component to the south-west (SW), connected by a bridge of $\mathrm{Ly} \alpha$ emission (hereafter Bifrost). Yggdrasil is visible in all four images, while Bifrost splits up into three components in the $235 \mathrm{GHz}$ observations (hereafter Freja, Thor and Odin). Thor is visible in the IRAC $3.6 \mu \mathrm{m}$ and $4.5 \mu \mathrm{m}$ images with an extension towards both Freja and Odin. A $\mathrm{CO}(8-7)$ emitting component (hereafter Loke) is located even further to the SW than Freja, Thor, and Odin. The complex morphology is illustrated by the schematic overview in Fig. 1 and additionally shows the positions of the radio lobes, and the $\mathrm{CO}(8-7)$ emission at the position of Yggdrasil and Loke. We now discuss each of these phases in detail. 

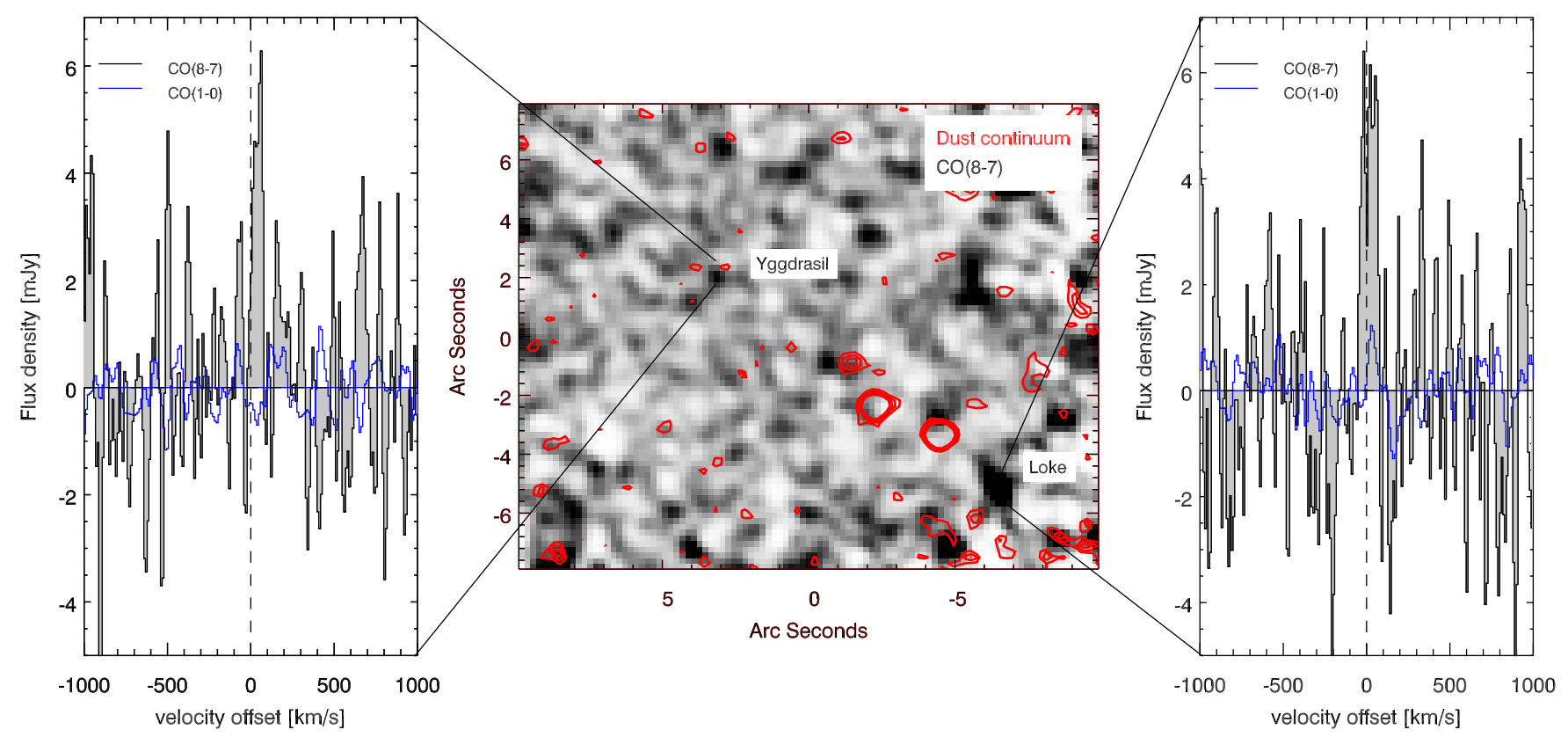

Fig. 2. ALMA cube showing $\mathrm{CO}(8-7)$ emission at two positions in the data cube: at the location of Yggdrasil and to the SW in an isolated component: Loke. Middle panel: the grey scale image of the $\mathrm{CO}(8-7)$ emission overlaid with the ALMA dust continuum contours. We note that the increase in noise towards the edges due to the primary beam correction. Left and right: the $\mathrm{CO}(8-7)$ velocity profiles for both Yggdrasil (left) and Loke (right). Surprisingly the $\mathrm{CO}(8-7)$ lines only have a small offset from the HeII systemic redshift. The spectra are extracted from a beam-sized area at the position of the emission, and have rmss of $1.5 \mathrm{mJy}$ and $1.0 \mathrm{mJy}$ respectively. Both detections are unresolved at the S/N of our data.

\subsection{Surprising dust and molecular gas distribution}

\subsubsection{Dust continuum emission}

Yggdrasil shows up in the ALMA submm observations as a weak dust continuum emission source at $235 \mathrm{GHz}$, with an extracted flux density over the region of $0.8 \pm 0.2 \mathrm{mJy}$ (see Table 4 ). However, the vast majority of the $235 \mathrm{GHz}$ dust emission originates from three components (Freja, Thor, and Odin) aligned along a "string" shifted $48-65 \mathrm{kpc}$ towards the SW relative to the position of Yggdrasil (see Fig. 1). Table 4 lists their flux densities extracted using CASA.

Considering the number counts of $>0.5 \mathrm{mJy}$ sources at $235 \mathrm{GHz}$ of $\sim 2 \times 10^{4} \mathrm{deg}^{-2}$ (Laurent et al. 2005), and the size of the area within the 8.' 7 radius from Yggdrasil to Odin, we expect to find 0.37 sources with $F_{235 \mathrm{GHz}}>0.5 \mathrm{mJy}$. We detect four sources within this area; the probability that these four sources are unrelated is therefore $0.37^{4}$, or $2 \%$. The probability that these four sources are associated to MRC0943-242 is thus 98\%.

\subsubsection{Molecular emission lines}

Searching the ALMA cube for molecular gas tracers, we find $\mathrm{CO}(8-7)$ emission at two different positions. One at the position of Yggdrasil and one $\sim 90 \mathrm{kpc} \mathrm{SW}$ of Yggdrasil. The $3.5 \sigma$ $\mathrm{CO}(8-7)$ detection at the position of Yggdrasil (see Fig.stemic redshift). Fitting a single Gaussian to this narrow velocity profile yields a FWHM of $47 \pm 13 \mathrm{~km} \mathrm{~s}^{-1}$, and a velocity integrated line flux of $0.33 \pm 0.06 \mathrm{Jy} \mathrm{km} \mathrm{s}^{-1}$. The other $3.5 \sigma \mathrm{CO}(8-7) \mathrm{de}-$ tection is located $\sim 90 \mathrm{kpc} \mathrm{SW}$ of Yggdrasil (Loke, see Figs. 1 and 2). Surprisingly, Loke is not detected in any dust continuum nor optical counter parts. Like the $\mathrm{CO}(8-7)$ line detected at the position of Yggdrasil, this $\mathrm{CO}(8-7)$ line is narrow with a FWHM of $53 \pm 17 \mathrm{~km} \mathrm{~s}^{-1}$, and only shifted by $\sim+16 \mathrm{~km} \mathrm{~s}^{-1}$ from the HeII systemic redshift (see Fig. 4). The rms of the $\mathrm{CO}(8-7)$ spectrum for Yggdrasil is $1.8 \mathrm{mJy}$ and $2.1 \mathrm{mJy}$ for Loke. This small difference is due to a combination of the primary beam correction (affecting only Loke) and continuum subtraction residuals (affecting only Yggdrasil, as no continuum is detected in Loke).

To verify the reality of the $\mathrm{CO}(8-7)$ emission, ensuring that these $\mathrm{CO}(8-7)$ lines are not rare but insignificant noise peaks in the cube, we create 13 moment- 0 maps at different frequencies in the cube by collapsing a velocity ranges in the cube of $180 \mathrm{~km} \mathrm{~s}^{-1}$. This corresponds to three times the average FWHM of two $\mathrm{CO}(8-7)$ lines and is the same velocity width as for the $\mathrm{CO}(8-7)$-moment-0 map (see Fig. 2). From these, we select the brightest areas in each moment- 0 map and extract the spectrum from the cube for each of these areas and ensure that there is no overlap between velocity regions. From this we find 81 unique peaks for which we extract over the full $233.5-236.5 \mathrm{GHz}$ frequency range. We fit a Gaussian profile to the brightest peak in each spectrum and determine the $\mathrm{S} / \mathrm{N}$ in each spectrum. Only two spectra show a peak of intensity with a $\mathrm{S} / \mathrm{N}$ of $\sim 3$ and a FWHM of $\sim 70 \mathrm{~km} \mathrm{~s}^{-1}$ which could resemble a real line. The chance of detecting a line such as Loke within $27 \pm 6 \mathrm{~km} \mathrm{~s}^{-1}$ from Yggdrasil within our $4000 \mathrm{~km} \mathrm{~s}^{-1}$ bandwidth is $27 / 4000=$ $0.67 \%$. In addition, the probability of finding a $\sim 50 \mathrm{~km} \mathrm{~s}^{-1}$ wide line like Yggdrasil and Loke is 50/4000 $=1.25 \%$. The combined probability of finding the observed $\mathrm{CO}(8-7)$ configuration in Loke and Yggdrasil is therefore only $0.84 \%$.

Searching the archival Australian Telescope Compact Array (ATCA) data for Yggdrasil (see Emonts et al. 2011 for more details about the data reduction and data characteristics) by reshifting the velocities to the redshift $(z=2.92296 \pm 0.00001$, see Sect. 3.2) used for this work, we find tentative $\mathrm{CO}(1-0)$ line emission at a $2.8 \sigma$ level at the position of Loke (see Fig. 2), consistent with the existence of a large molecular gas reservoir. The tentative detection of $\mathrm{CO}(1-0)$ emission and the $\mathrm{CO}(8-7)$ to $\mathrm{CO}(1-0)$ line flux ratio of $\sim 0.03$ would imply the presence of a low excitation molecular gas reservoir. $\mathrm{No} \mathrm{CO}(1-0)$ emission 
Table 1. Velocity integrated fluxes and FWHM of the resonance, fine-structure, and molecular lines for Yggdrasil, in-between Odin and Thor and Loke.

\begin{tabular}{|c|c|c|c|c|}
\hline \multicolumn{5}{|c|}{ MUSE } \\
\hline Line & $\begin{array}{l}\lambda_{\text {rest }} \\
\AA \\
\end{array}$ & $\begin{array}{l}\lambda_{\text {obs }} \\
\AA\end{array}$ & $\begin{array}{c}\text { Line flux } \\
10^{-16} \mathrm{erg} / \mathrm{cm}^{-2} / \mathrm{s} \\
\end{array}$ & $\begin{array}{l}F W H M \\
\mathrm{~km} \mathrm{~s}^{-1} \\
\end{array}$ \\
\hline \multicolumn{5}{|l|}{ Yggdrasil } \\
\hline $\operatorname{Ly} \alpha$ & 1215.7 & $4768.9 \pm 0.2$ & $26.60 \pm 0.25$ & $1592 \pm 44$ \\
\hline NV & $1238.8,1242.8$ & $4859.1 \pm 0.6,4875.5 \pm 0.9$ & $0.90 \pm 0.04$ & $1803 \pm 174$ \\
\hline SiIV & 1402.8 & $5500.1 \pm 1.1$ & $0.60 \pm 0.04$ & $2493 \pm 214$ \\
\hline NIV] & 1486.5 & $5830.3 \pm 1.9$ & $0.27 \pm 0.04$ & $1764 \pm 370$ \\
\hline CIV & $1548.2,1550.8$ & $6073.9 \pm 1.5,6072.2 \pm 1.4$ & $4.34 \pm 0.06$ & $1410 \pm 188$ \\
\hline HeII & 1640.4 & $6435.2 \pm 0.2$ & $3.04 \pm 0.05$ & $862 \pm 30$ \\
\hline OIII] & $1660.8,1666.1$ & $6516.3 \pm 1.4,6533.8 \pm 2.5$ & $0.50 \pm 0.03$ & $1492 \pm 292$ \\
\hline CIII] & $1906.7,1908.7$ & $7479.2 \pm 0.9,7490.9 \pm 3.1$ & $2.00 \pm 0.06$ & $1734 \pm 124$ \\
\hline $\mathrm{CII}]$ & 2326.0 & $9122.4 \pm 1.0$ & $0.90 \pm 0.04$ & $1606 \pm 118$ \\
\hline \multicolumn{5}{|l|}{ Bifrost } \\
\hline $\operatorname{Ly} \alpha$ & 1215.7 & $4769.2 \pm 1.1$ & $0.65 \pm 0.06$ & $1018 \pm 205$ \\
\hline \multicolumn{5}{|c|}{ ALMA } \\
\hline Line & $v_{\text {rest }}$ & $v_{\mathrm{obs}}$ & $S \mathrm{~d} V$ & FWHM \\
\hline & $\mathrm{GHz}$ & $\mathrm{GHz}$ & $\mathrm{Jy} \mathrm{km} \mathrm{s}^{-1}$ & $\mathrm{~km} \mathrm{~s}^{-1}$ \\
\hline \multicolumn{5}{|l|}{ Yggdrasil } \\
\hline $\mathrm{CO}(8-7)$ & 921.8 & $234.94 \pm 0.1$ & $0.33 \pm 0.07$ & $43 \pm 13$ \\
\hline \multicolumn{5}{|l|}{ Loke } \\
\hline $\mathrm{CO}(1-0)$ & 115.3 & - & $<0.08$ & $27 \pm 16$ \\
\hline $\mathrm{CO}(8-7)$ & 921.8 & $234.96 \pm 0.1$ & $0.54 \pm 0.10$ & $57 \pm 17$ \\
\hline \multicolumn{5}{|c|}{ Freja, Thor, and Odin } \\
\hline $\mathrm{CO}(8-7)$ & 921.8 & - & $<0.13$ & - \\
\hline $\mathrm{CO}(8-7)$ & 921.8 & - & $<0.18$ & - \\
\hline $\mathrm{CO}(8-7)$ & 921.8 & - & $<0.16$ & - \\
\hline
\end{tabular}

Notes. The ATCA observations have a beam size $11.5^{\prime \prime} \times 9.0^{\prime \prime}$ and PA $87.5^{\circ}$. The $3 \sigma$ upper limits on the $\operatorname{CO}(8-7)$ line emission for Freja, Thor, and Odin are given assuming a FWHM of $50 \mathrm{~km} \mathrm{~s}^{-1}$, i.e. similar to the $\mathrm{CO}(8-7)$ lines for Yggdrasil and Loke.

was found at the position of Yggdrasil (see Fig. 2). This agrees with the expectation that we should find more highly excited gas near a powerful radio-loud AGN (e.g. Weiß et al. 2007)

Unfortunately the ATCA observations are limited by the large beam and low $\mathrm{S} / \mathrm{N}$, and it is therefore not possible to determine whether or not $\mathrm{CO}(1-0)$ emission originates from the dust continuum sources, a free molecular gas component in the halo or both. In an attempt to explore if an extended $\mathrm{CO}(8-7)$ component is present, we applied a larger beam to the ALMA $235 \mathrm{GHz}$ data. Unfortunately, tapering the ALMA $235 \mathrm{GHz}$ data and convolving them with a beam comparable to that of ATCA did not produce useful results, as our shallow ALMA observation do not contain sufficient short baselines. We plan JVLA CO(1-0) and ALMA [CI] observations at arc-second spatial resolution to pinpoint the exact location and determine the characteristics of the molecular gas reservoir.

No $\mathrm{CO}(8-7)$ line emission is observed at the positions of Freja, Thor, and Odin. We therefore take the $3 \sigma$ upper limit of the line emission at these positions to be 3 times the rms of the spectra extracted with the same region sizes as for the continuum emission (see Table 1).

\section{2. lonised gas}

The MUSE spectrum of Yggdrasil (Fig. 3) detects a rich variety of ionised gas tracers. Overall, our spectrum is consistent with the Keck spectrum of Vernet et al. (2001), but now provides full spatial information over the large field $\left(1^{\prime} \times 1^{\prime}\right)$ of view of MUSE. Table 1 lists the velocity and integrated fluxes for the nine emission lines detected at the position of Yggdrasil. The flux is determined by integrating the spectral line profile within $3 \sigma$ of the noise. For our analysis, we adopt the redshift of HeII integrated over the peak of the continuum emission as the systemic velocity, as it is the brightest non-resonant line in our spectra. The HeII emission line is best fitted by a Lorentzian profile, with a centre corresponding to a redshift of $2.92296 \pm 0.00001$ and a FWHM of $862 \pm 30 \mathrm{~km} \mathrm{~s}^{-1}$. The flux within $3 \sigma$ is given in Table 1. The other high ionisation lines have profiles and redshifts similar to that of HeII (Table 1) and Fig. 4.

The Ly $\alpha(\lambda 1215.7 \AA)$ line is by far the brightest and most extended emission line in the MUSE data cube. It peaks at the position of the AGN, but extends out to $80 \mathrm{kpc}$ (see bottom panel of Fig. 5). Such extended Ly $\alpha$ haloes have been detected in several other HzRGs (e.g. van Ojik et al. 1996; Reuland et al. 2003; Swinbank et al. 2015). The Ly $\alpha$ halo in MRC0943-242 shows a linear filament, Bifrost, connecting Yggdrasil with the two brightest dust continuum components (Thor and Odin), strongly suggesting that they are physically connected (see Fig. 5). The peak intensity of Bifrost is $\sim 18$ times fainter than that for the Ly $\alpha$ detection of Yggdrasil (see panels A and D of Fig. 5).

The Ly $\alpha$ emission on the nucleus exhibits four absorption features in our data. We denote these absorption features as 1,2, 3 , and 4 where the bluest feature is 1 , middle are 2 and 3 , and the reddest absorption feature 4 (in accordance with the labelling used by Jarvis et al. 2003, see panel A of Fig. 5). Furthermore, component 2 is related to the strong CIV absorption observed against the nucleus (Binette et al. 2000). By centring the Ly $\alpha$ velocity profile at the fitted central velocity of HeII we find that the bottom of the absorption trough is at a redshift off $z_{\mathrm{abs}}=$ $2.91864 \pm 0.00002$. By fitting a Voigt-profile to the absorption 


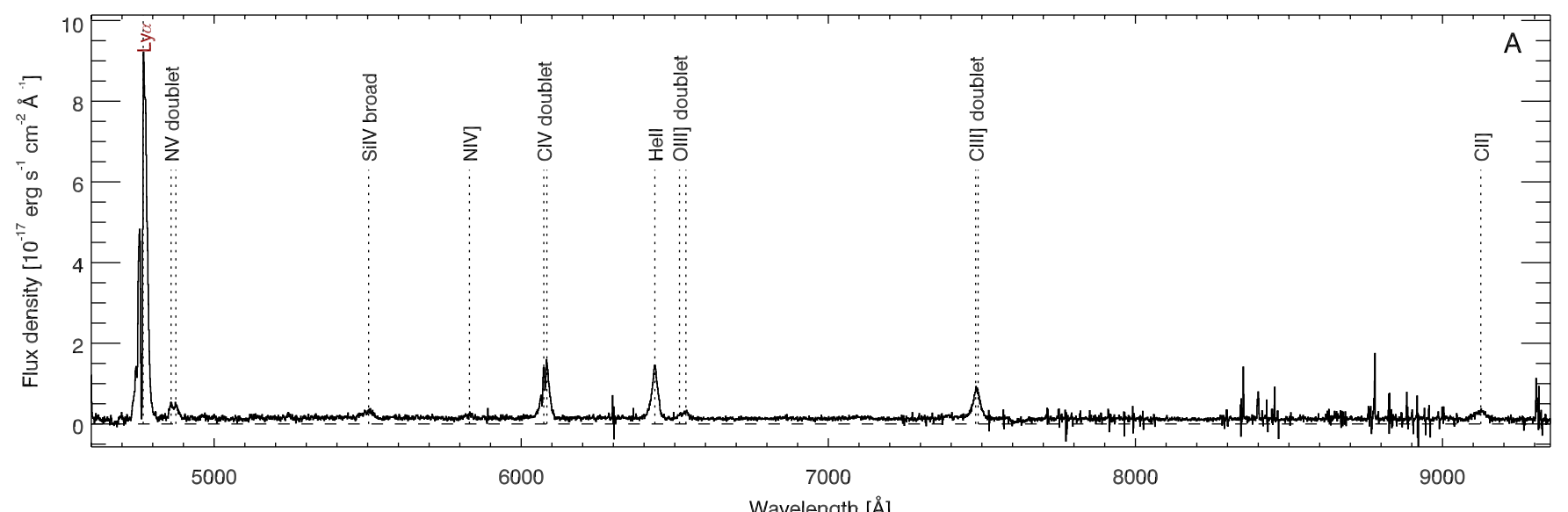

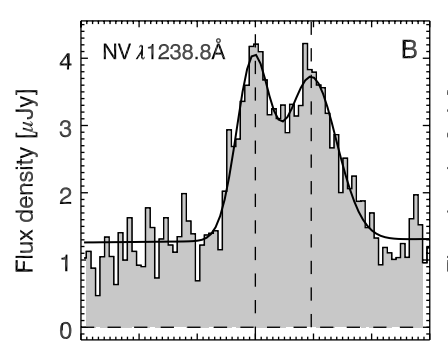

$-3000-2000-1000 \quad 0 \quad 100020003000$ Velocity offset $[\mathrm{km} / \mathrm{s}]$

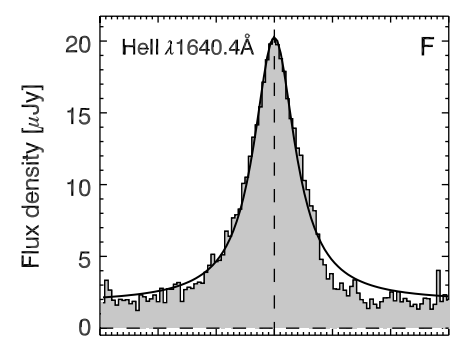

$-3000-2000-1000 \quad 0 \quad 100020003000$

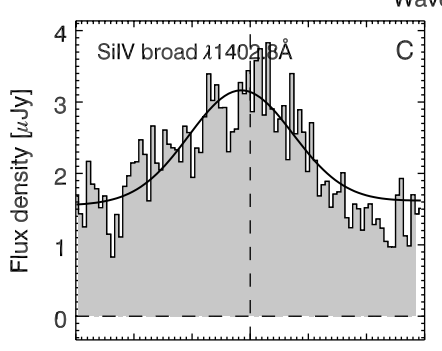

-3000-2000-1000 0 100020003000 Velocity offset [km/s]

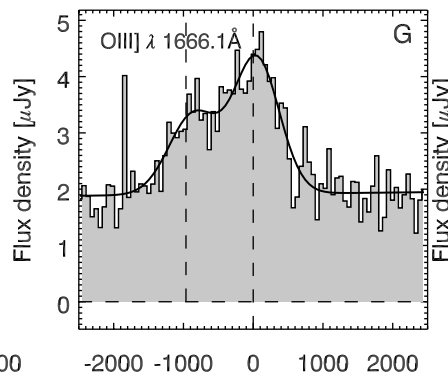

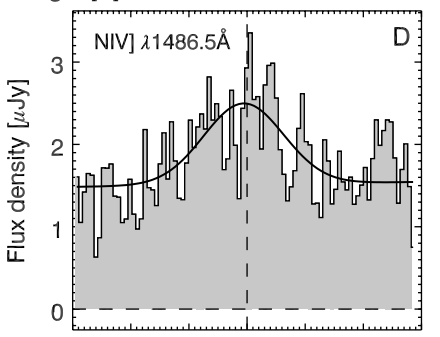

-3000-2000-1000 0 100020003000 Velocity offset $[\mathrm{km} / \mathrm{s}]$

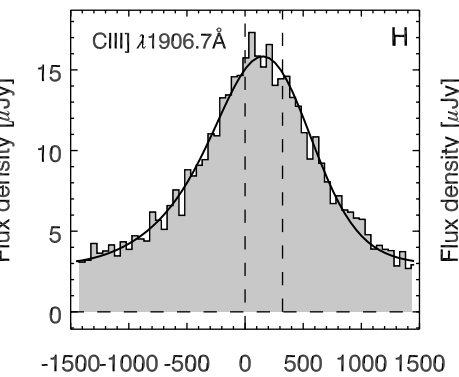

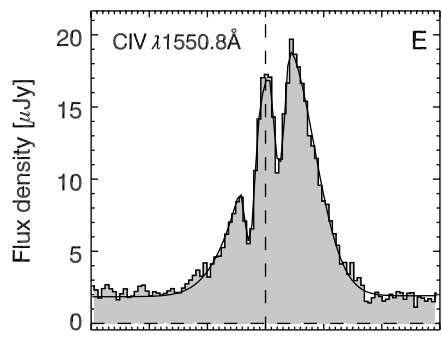

-3000-2000-1000 $0 \quad 100020003000$ Velocity offset $[\mathrm{km} / \mathrm{s}]$

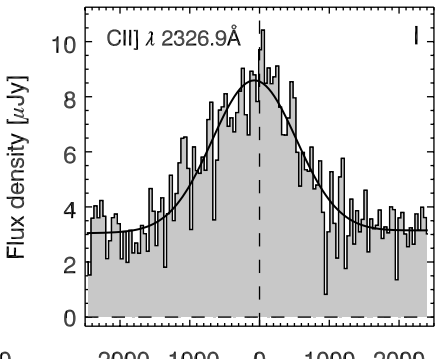

Fig. 3. Panel A): full MUSE spectrum for Yggdrasil. All detected lines (other than Ly $\alpha$ ) are indicated with a black dotted lines. Panels B)-I): velocity profiles for NV, SiIV, NIV], CIV, HeII, OIII],CIII], and CII] assume HeII as the systemic redshift. The CIV emission line shows absorption troughs like those of the Ly $\alpha$ emission line.

trough, we estimate a column density of the neutral gas for component 2 of $N_{\mathrm{HI}}=1.3 \times 10^{19} \mathrm{~cm}^{-2}$ and a Doppler width of $64 \mathrm{~km} \mathrm{~s}^{-1}$ (see Table 2) which are comparable to the values determined by Binette et al. 2000. We note that because the line is saturated, this estimate is very uncertain and likely a lower limit to the true value. For components 1,3 , and 4 , we find good agreement with the values in Jarvis et al. (2003) for the column densities and velocities.

We observe two absorption troughs in the CIV emission line; Voigt profile fitting to the two features reveals that they are caused by gas on the line of sight at redshift $z_{\text {abs }}=2.91940 \pm$ 0.00007 and $2.91945 \pm 0.000061$ and column densities of $N_{\mathrm{CIV}}=$ $3.4 \times 10^{14} \mathrm{~cm}^{-2}$ and $=6.9 \times 10^{14} \mathrm{~cm}^{-2}$, higher than $3.2 \times 10^{14} \mathrm{~cm}^{-2}$ found by Binette et al. (2000).

Stepping through the cube towards increasing wavelengths reveals the complexity of the emission in the MRC0943-242 system (see Figs. 5-7):

- $\lambda_{\text {obs }} \sim 4732-4747 \AA$ : Considering the Ly $\alpha$ emission line profile at Yggdrasil (see panel A of Fig. 5); the emission furthest to the blue side of the line profile is spatially centred around the radio source and approximately within the region containing the radio emission (see also panel A Fig. 6).
Table 2. Parameters determined by fitting a Voigt profiles to the absorption troughs.

\begin{tabular}{lccc}
\hline \hline Line & $\begin{array}{c}N_{\mathrm{HI}} \\
\mathrm{cm}^{-2}\end{array}$ & $\begin{array}{c}b \\
\mathrm{~km} \mathrm{~s}^{-1}\end{array}$ & $z_{\mathrm{abs}}$ \\
\hline Yggdrasil & & & \\
Ly $\alpha$ absorber 1 & $5.0 \times 10^{13}$ & 84 & $2.90689 \pm 0.00032$ \\
Ly $\alpha$ absorber 2 & $1.3 \times 10^{19}$ & 64 & $2.91864 \pm 0.00002$ \\
Ly $\alpha$ absorber 3 & $3.7 \times 10^{13}$ & 140 & $2.92641 \pm 0.00023$ \\
Ly $\alpha$ absorber 4 & $1.7 \times 10^{13}$ & 33 & $2.93254 \pm 0.00007$ \\
CIV absorber 2 & $3.4 \times 10^{14}$ & 94 & $2.91940 \pm 0.00007$ \\
& $6.9 \times 10^{14}$ & 100 & $2.91945 \pm 0.00006$ \\
\hline
\end{tabular}

\section{Bifrost}

Ly $\alpha$ absorber $2 \quad 7 \times 10^{18}$

$2.91841 \pm 0.00045$

Notes. Fits are performed on the absorption troughs in the Ly $\alpha$ line observed for both Yggdrasil and in-between Odin and Thor. The CIV line has been fitted with two Voigt profiles with the same fitting parameters.

- $\lambda_{\text {obs }} \sim 4747-4754 \AA$ : As we move towards the red, over the wavelength range of absorption feature 1 , we see strong nuclear emission. 


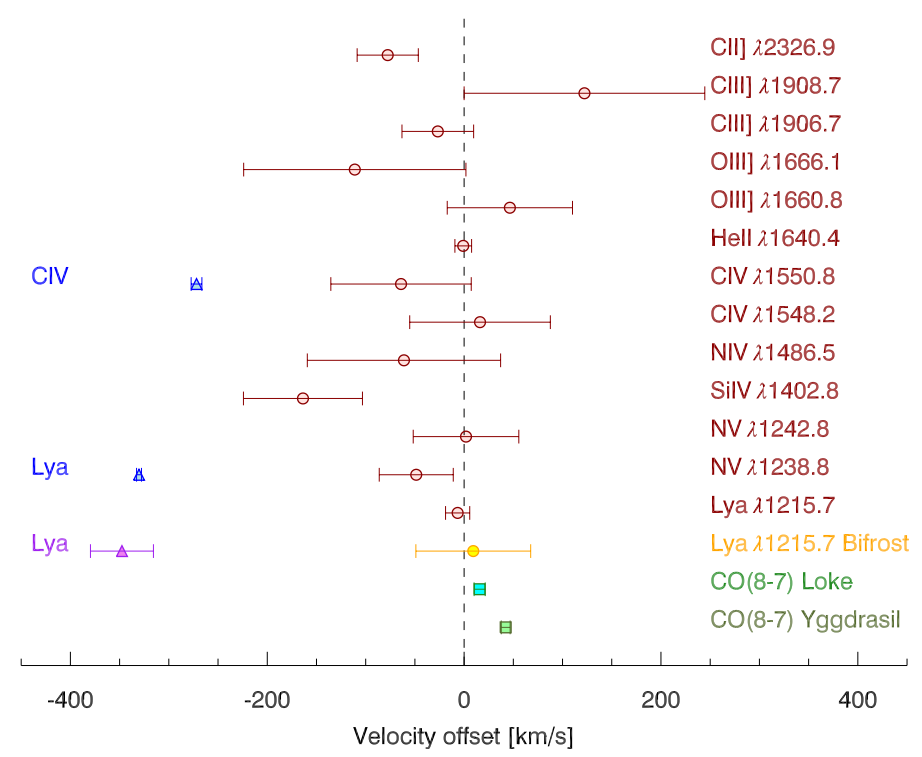

Fig. 4. Offset of the emission line and absorption features centres from the HeII systemic redshift. Red points are emission lines observed for Yggdrasil. Yellow is the $\operatorname{Ly} \alpha$ emission line for Bifrost. Green is the $\mathrm{CO}(8-7)$ emission lines for Yggdrasil and Loke. Blue and purple are the centre of the absorption feature 2 . The emitting components are distributed within $\pm 200 \mathrm{~km} \mathrm{~s}^{-1}$ of the systemic velocity, while the absorbers are all blue shifted by $\sim 350 \mathrm{~km} \mathrm{~s}^{-1}$.

$-\lambda_{\text {obs }} \sim 4754-4759 \AA$ : Moving further to the red between absorption features 1 and 2, we observe a closed region of bubble-like emission reaching $\sim 20 \mathrm{kpc}$ out, surrounding the western radio lobe (see panel B of Fig. 6). The absorption components 1 and 2 are visible against this emission across the region of Yggdrasil (see panels A-C of Fig. 5).

- $\lambda_{\text {obs }} \sim 4759-4769 \AA$ : As we step further to the red, through the wavelength range of absorption feature 2, the Ly $\alpha$ emission becomes fainter - down to our surface brightness detection limits. We note that this is in agreement with the Ly $\alpha$ profiles of all the extended emission - when we have sufficient surface brightness to have detectable line emission, we see absorption component 2 which has zero intensity in the line core. This includes the Ly $\alpha$ emission from Bifrost (see panel D in Fig. 5) and the CIV emission from Yggdrasil. The absorption component 2 in Bifrost is at a redshift of $z_{\mathrm{abs}}=2.91841 \pm 0.00045$, consistent with that in Yggdrasil (see panel D of Fig. 5). This suggests that the absorption feature in the spectrum of Bifrost is due to absorption component 2, meaning that absorber 2 has an extent of at least $65 \mathrm{kpc}$ from Yggdrasil. The data suggest a lower column density of absorber 2 in Bifrost of $N_{\mathrm{HI}} \sim 7 \times 10^{18} \mathrm{~cm}^{-2}$. We warn that this $N_{\mathrm{HI}}$ is particularly uncertain due to the low $\mathrm{S} / \mathrm{N}$ of the data, and the degeneracy between $N_{\mathrm{HI}}$ and the bparameter. Deeper and higher resolution data are required to trace column density variations across the system. We also note that in this wavelength range, we observe high ionisation emission lines of HeII, CIV, and CIII] from gas extending over $7^{\prime \prime}$ or $\sim 60 \mathrm{kpc}$ to the west (see panel $\mathrm{F}$, the green contours of the central panel of in Fig. 5 and the CIV and HeII moment-0 maps in panels A and B of Fig. 7). Fainter, less extended emission is also observed to the east of the nucleus. Deeper data will be needed to determine the exact distribution of absorption component 2; however, since the line core is black throughout the extend of the cube, we can conclude that it is extended with a unity covering factor.

- $\lambda_{\text {obs }} \sim 4769-4776 \AA$ : At wavelengths to the red of absorption feature 2, and containing the absorption feature 3 (see red contours of Fig. 5 and panel $\mathrm{C}$ in Fig. 6), we observe the emission from Bifrost. Where the Ly $\alpha$ emission is sufficiently bright, we see absorption component 3 superposed on the line emission (see panels A-C of Fig. 5).

- $\lambda_{\text {obs }} \sim 4779-4794 \AA$ : Further to the red - at longer wavelengths than the range of absorption feature 3, component 4 shows up as a small trough (see panels A-C of Fig. 5 and panel D of Fig. 6) before we again start to see circum nuclear emission all contained within the radius encompassing the radio lobes.

- $\lambda_{\text {obs }} \sim 6025-6125$ and 6422-6430 ̊: Moving to wavelengths of the CIV and HeII emission, we do not have sufficient $\mathrm{S} / \mathrm{N}$ to split these lines into velocity channels like for Ly $\alpha$. Instead, we present line maps of the central parts of these lines in panels A and B of Fig. 7. Both lines display a similar morphology, which is also seen (albeit at lower $\mathrm{S} / \mathrm{N}$ ) in CIII] (panel C of Fig. 7). We detect a tail of CIV and HeII emission to the WSW (panels A and B of Fig. 7); however this tail is not spatially aligned with the Ly $\alpha$ bridge (Bifrost) connecting Yggdrasil with Freja, Thor, and Odin. Interestingly, the CIV and HeII tail is not seen in Ly $\alpha$ (see the blue contours of the middle panel and panel E of Fig. 5). Fitting a Lorentz profile to this HeII emission line as well yields a redshift of $z=2.916$ which is consistent with the emission being at the same velocity offset as absorption component 2. This suggest that this CIV and HeII emitting gas is also seen in Ly $\alpha$, but that this is absorbed completely by component 2.

The large wavelength coverage of MUSE allows us to detect other fine structure lines (see panels B-I of Fig. 3). The three doublets NV, OIII], and CIII] are well detected at a $\geq 6 \sigma$ level. The NV doublet is spatially resolved and reveals two spectral lines of similar intensity. The OIII] doublet is blended, but there is some indications of the doublet structure with the $\lambda 1660.8 \AA$ line being a shoulder in the blue side of the OIII] $\lambda 1666.1 \AA$ line. We also detect a broad SiIV component, a weak NIV] line at a $2.3 \sigma$ level, and a CII] line at a $5.5 \sigma$ level at the edge of the band. The CII] emission line is a blend of multiple lines, which explains why the line peaks on the red side of $\lambda 2326 \AA$. Table 1 lists the line parameters for these fine structure lines over the circum nuclear region (see Fig. 3).

All the emission from NV, SiIV, NIV], OIII], and CII] lies within the region encompassed by the radio lobes. The CII] emission is a particularly spectacular example as it delineates both the extension and direction of the radio jet and lobes (see panel D of Fig. 7).

\subsection{Stellar mass}

The wavelengths covered by Spitzer probe the rest-frame nearand mid-IR part of the spectrum, which is believed to be the best tracer of the stellar-mass with almost no contributions from the thermal emission from the AGN (Seymour et al. 2007). Yggdrasil has a well sampled SED with detections at $3.6 \mu \mathrm{m}$, $4.5 \mu \mathrm{m}, 8.0 \mu \mathrm{m} 16 \mu \mathrm{m}, 24 \mu \mathrm{m}$, and upper limits for $5.8 \mu \mathrm{m}$, $70 \mu \mathrm{m}$, and $160 \mu \mathrm{m}$. Seymour et al. (2007) and De Breuck et al. (2010) fit a toy model to these points, composed of four components: an elliptical galaxy composite stellar population (CSP) and three blackbody (BB) components with temperatures 


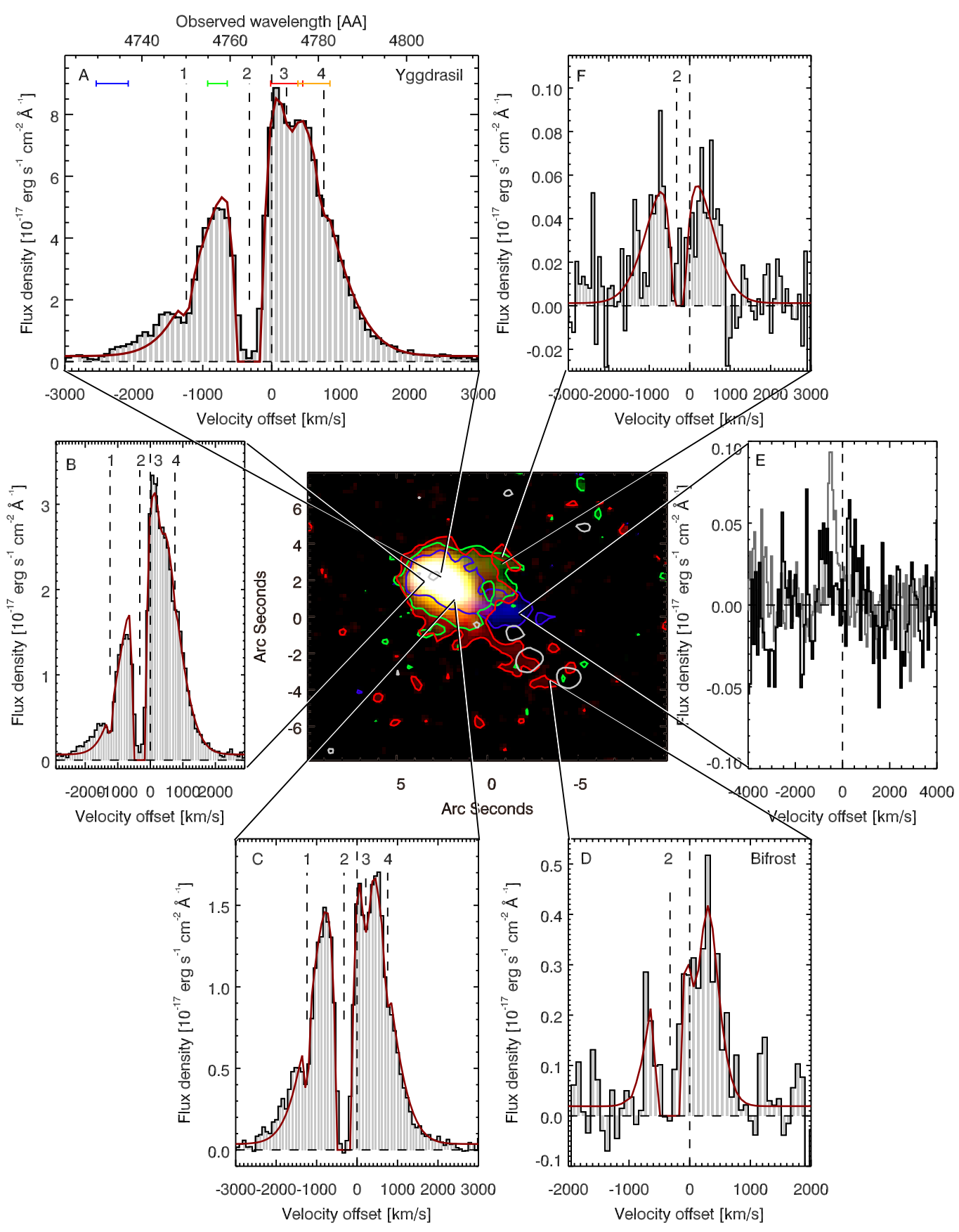

Fig. 5. Overview of emission and absorption components at different positions around MRC0943-242. Middle Panel: composite red-green-blue image of Ly $\alpha$ and HeII emission (see caption of Fig. 1 for more details) with the ALMA $235 \mathrm{GHz}$ contours overlaid in grey. Panel A): the Ly $\alpha$ line profile of the full area of Yggdrasil (elliptical aperture of $1.3^{\prime \prime} \times 0.7^{\prime \prime}$ ), showing all four absorption components. Component 2 is the most prominent and goes to zero intensity at its centre. The spectrum has an rms of $0.3 \times 10^{-17} \mathrm{erg} \mathrm{s}^{-1} \mathrm{~cm}^{-2} \AA^{-1}$. The blue, green, red, and orange bars above the spectrum shows the range in wavelength the channelmaps in Fig. 6 have been summed over. The blue correspond to panel A), green to panel B), red to panel $\mathbf{C}$ ), and orange to panel $\mathbf{C}$ ) in Fig. 6. Panels B) and C): the Ly $\alpha$ profiles of two areas near the nucleus (circular 0.5" apertures) and have rms of $0.1 \times 10^{-17} \mathrm{erg} \mathrm{s}^{-1} \mathrm{~cm}^{-2} \AA^{-1}$. These profiles likewise show signs of all four absorption components. Panel D): the Ly $\alpha$ profile of Bifrost (circular 1.2" aperture and rms of $0.1 \times 10^{-17} \mathrm{erg} \mathrm{s}^{-1}$ ) showing sign of absorption component 2. Panel E): spectrum extracted at the HeII emitting tail ( $0.7^{\prime \prime}$ aperture), showing no sign of Ly $\alpha$ emission and an rms of $0.02 \times 10^{-17} \mathrm{erg} \mathrm{s}^{-1} \mathrm{~cm}^{-2} \AA^{-1}$. The HeII lines is over-plotted in grey. Panel F): the Ly $\alpha$ profile of the western extended Ly $\alpha$ emitting gas (0.4" aperture). All Ly $\alpha$ velocity profiles have been fitted with Voigt profiles superimposed on a Gaussian profile. These fits are shown as the red curves over plotted on the spectra.

of $60 \mathrm{~K}, 250 \mathrm{~K}$ and between $500-1500 \mathrm{~K}$ (for more details see Seymour et al. 2007 and De Breuck et al. 2010). This SED fitting results in an $H$-band stellar luminosity of $L_{H}^{\text {stellar }}=2.5 \times 10^{11} L_{\odot}$, which is converted to a stellar mass of $1.7 \times 10^{11} M_{\odot}$ by assuming a dust-free, passively evolving elliptical galaxy which started its formation at $z=10$ with no recent episodes of starformation. The SED allows for a good decomposition of the stellar and hot-dust dominated components, so the derived stellar 
B. Gullberg et al.: The mysterious morphology of MRC0943-242 as revealed by ALMA and MUSE
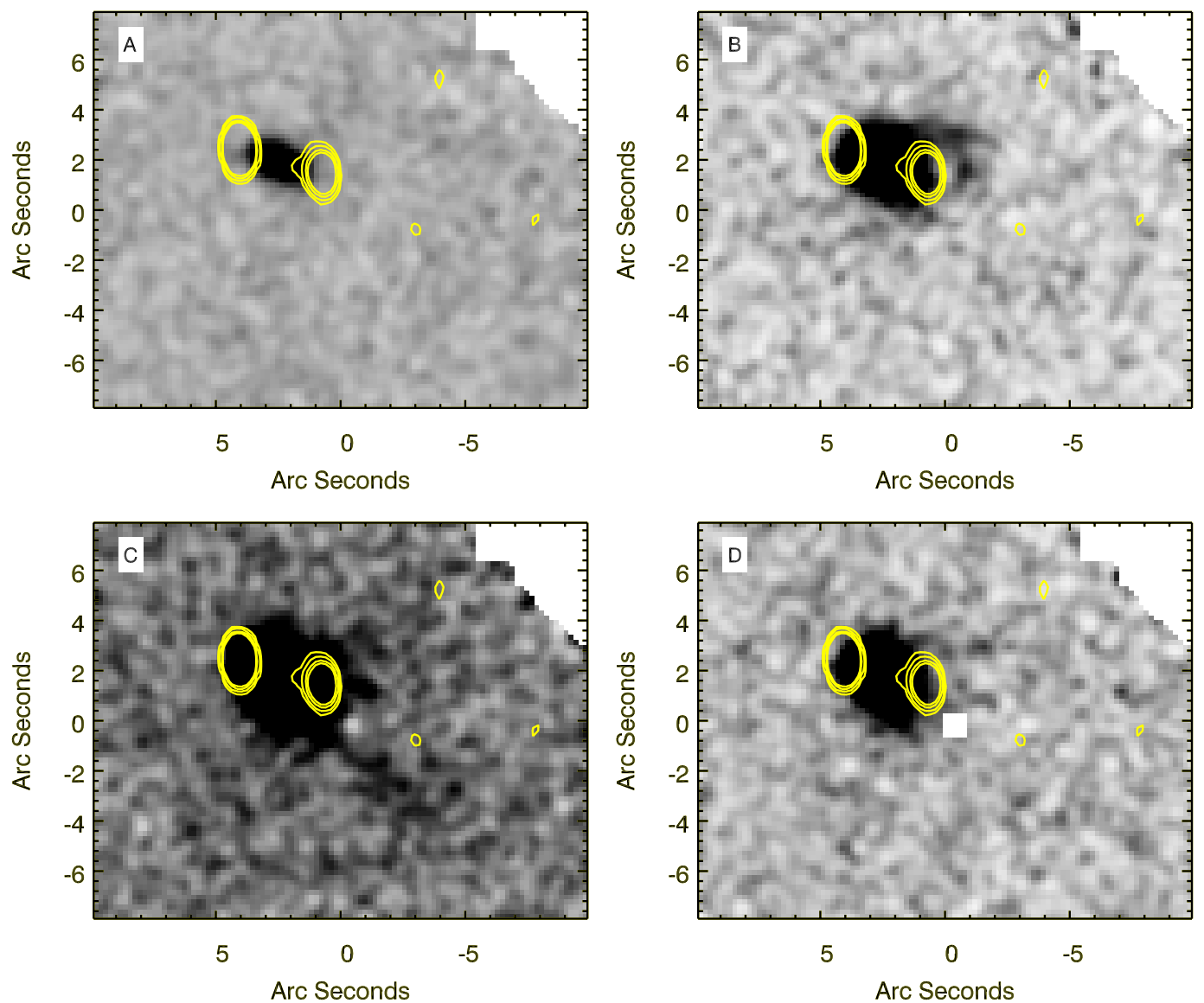

Fig. 6. Ly $\alpha$ Moment-0 maps summed over the wavelength ranges illustrated with the coloured bars in panel A) of Fig. 5: Panel A): sum over the blue bar $\left(\lambda_{\mathrm{obs}}=4732.5-4747.5\right)$. Panel B): sum over the green bar $\left(\lambda_{\mathrm{obs}}=4754.3-4758.8\right)$. Panel C): sum over the red bar $\left(\lambda_{\mathrm{obs}}=4768.8-4776.2\right)$. Panel D): sum over the orange bar $\left(\lambda_{\mathrm{obs}}=4778.8-4793.8\right)$.

Table 3. Photometric IRAC and MIPS points for Yggdrasil, Thor, and Loke after de-blending.

\begin{tabular}{ccccc}
\hline \hline Instrument & $\begin{array}{c}\text { Wavelength } \\
\mu \mathrm{m}\end{array}$ & $\begin{array}{c}S_{\text {Yggdrasil }} \\
\mu \mathrm{Jy}\end{array}$ & $\begin{array}{c}S_{\text {Thor }} \\
\mu \mathrm{Jy}\end{array}$ & $\begin{array}{c}S_{\text {Loke }} \\
\mu \mathrm{Jy}\end{array}$ \\
\hline IRAC 1 & 3.6 & 21.9 & 9.02 & $<2.10$ \\
IRAC 2 & 4.5 & 36.1 & 21.0 & $<1.32$ \\
IRAC 3 & 5.8 & - & - & - \\
IRAC 4 & 8.0 & 30.2 & $<21.4$ & - \\
MIPS & 24 & $468 \pm 40$ & $180 \pm 40$ & - \\
\hline
\end{tabular}

Notes. Thor is not detected in the IRAC 3 image, however the flux at the position is influenced by an image artefact from a nearby star in the field. Extracting even an upper limit at this position is therefore not possible. Loke is not detected in any of the IRAC images; we quote the $3 \sigma$ upper limits (Wylezalek et al. 2013).

mass is expected to be reliable (see Seymour et al. 2007, for a more detailed discussion on the uncertainties).

Examining the IRAC $3.6 \mu \mathrm{m}$ and $4.5 \mu \mathrm{m}$ image again, now with the knowledge of the presence of Freja, Thor, and Odin, we find stellar emission at the position of Thor, with an extension towards Freja and Odin (see Fig. 1 top left and Table 3). Also for Thor are the stellar and hot-dust emission well separated in the SED. We scale the stellar mass found for Yggdrasil, to the emission seen in the IRAC $4.5 \mu \mathrm{m}$ map (Table 6), yielding a stellar mass of $1.0 \times 10^{11} M_{\odot}$. For Loke, we accordingly derive an upper limit to the stellar mass of $\lesssim 6.2 \times 10^{9} M_{\odot}$.
Table 4. Positions and $235 \mathrm{GHz}$ fluxes for all components of MRC0943-242.

\begin{tabular}{|c|c|c|c|}
\hline \multirow{2}{*}{ Component } & \multicolumn{2}{|c|}{ Position } & \multirow{2}{*}{$\begin{array}{c}S_{235 \mathrm{GHz}} \\
\mathrm{mJy}\end{array}$} \\
\hline & RA & Dec & \\
\hline Yggdrasil & $09: 45: 32.769$ & -24.28 .49 .29 & $0.8 \pm 0.2$ \\
\hline Odin & $09: 45: 32.222$ & -24.28 .55 .06 & $2.0 \pm 1.0$ \\
\hline Thor & $09: 45: 32.386$ & -24.28 .54 .05 & $1.9 \pm 0.8$ \\
\hline Freja & $09: 45: 32.445$ & -24.28 .52 .55 & $0.6 \pm 0.3$ \\
\hline Loke & $09: 45: 32.072$ & -24.28 .56 .94 & $<0.3$ \\
\hline
\end{tabular}

Notes. As no dust continuum is observed for the position of Loke we infer a $3 \sigma$ upper limit of the $235 \mathrm{GHz}$ of three times the rms. The uncertainty includes the $15 \%$ flux calibration error.

\subsection{Disentangling the SED}

Yggdrasil has a well-sampled SED with IRAC, MIPS, SPIRE $(250 \mu \mathrm{m}, 350 \mu \mathrm{m}$, and $500 \mu \mathrm{m})$, and PACS $(24 \mu \mathrm{m}, 100 \mu \mathrm{m}$ and $160 \mu \mathrm{m}$ ) from previous studies (De Breuck et al. 2010; Drouart et al. 2014). The PACS $160 \mu \mathrm{m}$ image (see Appendix C of Drouart et al. 2014) reveals an elongated source, spanning from the position of Yggdrasil towards Freja, Thor, and Odin. Likewise, when returning to the IRAC $3.6 \mu \mathrm{m}$ and $4.5 \mu \mathrm{m}$ images (see panels A and B of Fig. 1), multiple components show up corresponding to Yggdrasil and Thor (see Sect. 3.3). While these sources can be separated in the Spitzer data, they are blended in the Herschel data. However, the high spatial resolution and sensitivity of the ALMA data allows us to disentangle 

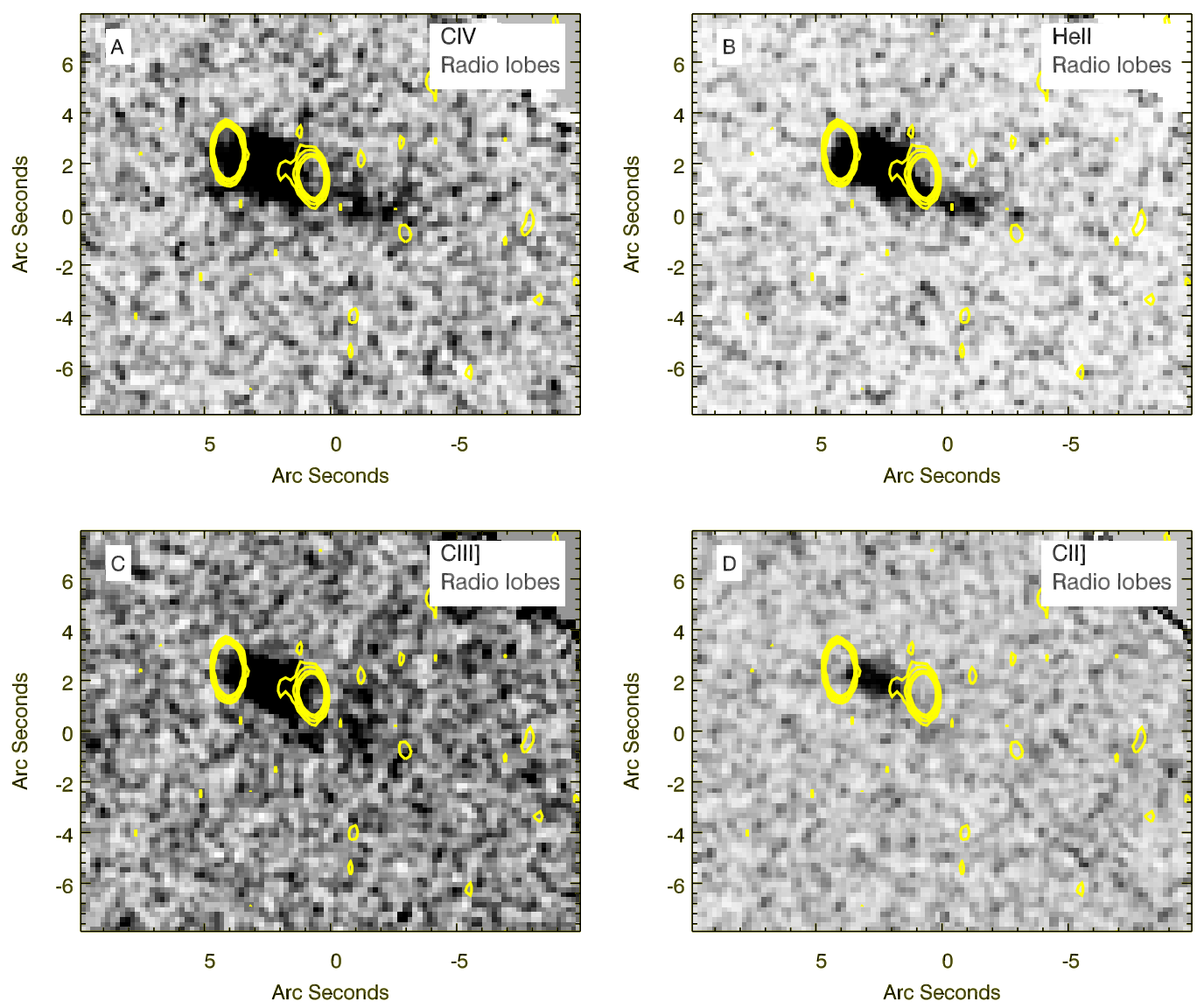

Fig. 7. Continuum subtracted moment-0 maps of the CIV, HeII, CIII], and CII] emission lines. The continuum is determined from the nearby line free channels for each line. Panel A): sum over the CIV emission line for the wavelength range $\lambda_{\mathrm{obs}}=6050-6090 \AA ̊$. Panel B): sum over the HeII emission line for the wavelength range $\lambda_{\mathrm{obs}}=6422-6430 \AA$ and is the same image as the blue colour of the middle panel of Fig. 5. Panel C): sum over the CIII] emission line for the wavelength range $\lambda_{\mathrm{obs}}=7455-7485 \AA$. Panel D): sum over the CII] emission line for the wavelength range $\lambda_{\text {obs }}=9071-9171 \AA$ A . Over-plotted with yellow contours in all panels is the VLA radio map, as a reference.

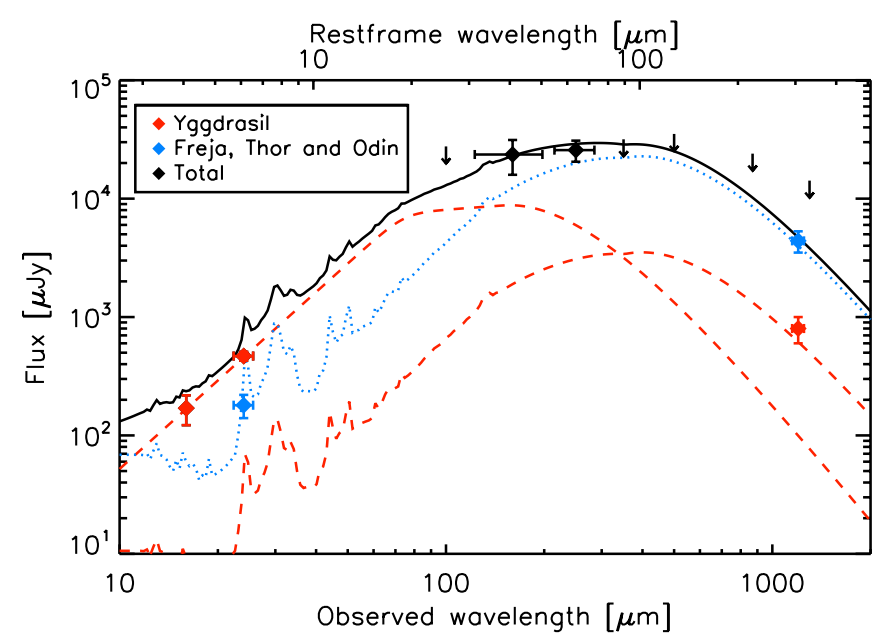

Fig. 8. Spectral energy distribution of MRC0943-242. The spatial resolution of the ALMA data allows us to disentangle the SED in the AGN heated component (red curve) and star formation heated component (blue curve). The sum of these two components is illustrated by the black curve.

the AGN and star formation heated components in the SED at $235 \mathrm{GHz}$. We conclude that the star-formation, as probed by the
Table 5. Infrared luminosities of each component, determined from our SED presented in Fig. 8.

\begin{tabular}{lcc}
\hline \hline Component & $L_{\mathrm{IR}}$ & Perc. \\
& {$\left[10^{12} L_{\odot}\right]$} & \\
\hline Total (Yggdrasil+Freja+Thor+Odin) & 17.2 & 100 \\
Freja+Thor+Odin (SB) & 8.4 & 49 \\
Yggdrasil (AGN) & 7.5 & 43 \\
Yggdrasil (SB) & 1.3 & 8 \\
\hline
\end{tabular}

dust emission, is taking place at a distance of $48-65 \mathrm{kpc}$ from the AGN in three smaller aligned components (Freja, Thor, and Odin). Table 3 lists the de-blended MIPS and IRAC photometry points.

Relying on the method of Drouart et al. (2014) by fitting an SED with AGN and starburst components, we now go further, and use the sub-arcsec resolution provided by the new dust continuum image to further disentangle the relative contribution of AGN and star formation spatially. Figure 8 shows the composite SED of the system, the three components being two spatially distinct positions with i) Yggdrasil ii) and Freja, Thor, and Odin photometry as red and blue points respectively. The unresolved far-IR Herschel detections and upper limits are shown as black points. It is important to note that each component is fitted individually, and that the black points are not used 
in the fit. However, the total SED and unresolved data (in black) provide a strong constraint on the total integrated flux from all components; all solutions where the sum is inconsistent with the Herschel photometry are excluded. In practice, the starburst component in Freja, Thor, and Odin is well constrained (blue dotted line in Fig. 8). However, the AGN component cannot simultaneously fit both the Spitzer and ALMA photometry without exceeding the Herschel photometry. We are therefore forced to add an additional starburst component to fit the Yggdrasil ALMA point. However, with only a single point, the starburst luminosity is only loosely constrained.

We clearly see from the SED fitting that Thor is starformation dominated, with the MIPS $24 \mu \mathrm{m}$ and $235 \mathrm{GHz}$ reproduced by the starburst model from DecompIR (Mullaney et al. 2011). Yggdrasil represents a composite of emission from an AGN and star formation as reported earlier (Drouart et al. 2014). However, the relative contribution to the IR luminosity from the AGN and the star formation is different; favouring the AGN as the main contributor. In fact, the AGN emits $\sim 40 \%$ of the IR luminosity of the entire system $\left(L_{\text {total }}^{\mathrm{IR}}=1.7 \times 10^{13} L_{\odot}\right)$, the remaining $\sim 60 \%$ is star-formation split between i) Yggdrasil and ii) Freja, Thor, and Odin at $\sim 10 \%$ and $\sim 50 \%$ levels (Table 5). According to the Kennicutt (1998) relation, Yggdrasil and Freja, Thor and Odin have SFR of $\sim 200$ and $\sim 1400 M_{\odot} \mathrm{yr}^{-1}$, respectively. Which, in turn, corresponds to a sSFR of $\sim 10^{-9}$ and $10^{-8} \mathrm{yr}^{-1}$.

\section{Discussion}

The wealth of information provided by the MUSE and ALMA data, even with rather modest integration times for both sets of data, paints a complicated picture from the warm ionised gas to the cold molecular gas and dust. We now discuss how the mass is distributed in each component and what the relationship between the components is.

\subsection{The nature of the gas and dust}

The MRC0943-242 system has a complex distribution of the ionised and neutral gas. The high ionisation lines are distributed mainly around the nucleus and are confined within the radio lobes. The exception to this is the extended HeII, CIV, and CIII] emission which is most extended to the west - beyond the western radio lobe (see panels A-C of Fig. 7). The fact that metals are detected within this part of the halo suggests the gas is not pristine, while the high ionisation state of the gas is consistent with photoionisation by the AGN, indicating that there is a cone of ionisation along the direction of the radio jet but extending well beyond the radio lobes. The lack of very extended Ly $\alpha$ emission in the outer region of the ionisation cone is most likely caused by HI gas distributed over large scales absorbing the emission (absorption component 2). The inner regions of the ionisation cone, i.e. the region that lies within or just beyond the radio lobes, shows significant $\mathrm{Ly} \alpha$ emission (and also absorption from component 2). Resonance scattering shifts the frequency of the Ly $\alpha$ photons and allow them to "leak" out on both sides of the absorption feature and broaden the line. In fact, throughout the region of Yggdrasil, we see all absorption components either in all places (as for components 1,2, and 3) or in particular regions (as for component 4). Since the absorption features are only rarely reaching zero intensity in their cores, the neutral gas responsible for absorption systems 1,3 , and 4 are mixed with Ly $\alpha$ line emitting regions (although some of it overlies the Ly $\alpha$ emission). Absorption component 2 must cover the full Ly $\alpha$ emitting region, including that out to $\sim 60 \mathrm{kpc}$, which is the projected distance to Bifrost. Since this absorption contains no flux in the line core (it is dark), the absorber must have unity covering factor and be optically thick. This being the case, our estimate of the HI column density is a lower limit.

This situation is somewhat akin to the large extended Ly $\alpha$ absorber on the line of sight to the HzRG TN J1338-1942 at $z=4.1$ (Swinbank et al. 2015). In this case the neutral gas reservoir extends at least $150 \mathrm{kpc}$ away from the core of the $\mathrm{H}_{z} \mathrm{RG}$. To detect this extended emission, Swinbank et al. (2015) obtained a much deeper integration and hence significantly more extended Ly $\alpha$ emission and absorption. However, unlike the deepest absorption feature in TN J1338-1942, the offset velocity of the absorption component 2 in MRC0943-242, is rather more modest, only a few $100 \mathrm{~km} \mathrm{~s}^{-1}$ compared to $1200 \mathrm{~km} \mathrm{~s}^{-1}$. Deeper data for MRC0934-242 may reveal a greater extent and more complex structure of the emitting and absorbing gas.

In addition to the ionisation cone and the general distribution of the Ly $\alpha$ emission around the central regions of Yggdrasil $\left(M_{*} \simeq 1.7 \times 10^{11} M_{\odot}\right)$, there is a bridge of material connecting the IRAC continuum emission of Thor and beyond $\left(M_{*} \simeq\right.$ $\left.1.0 \times 10^{11} M_{\odot}\right)$. Ivison et al. (2008) also found multiple components for the $\mathrm{HzRG} 4 \mathrm{C} 60.07$ at $z=3.8$, likewise connected by a bridge of gas. They interpret this bridge as a plume of cold dust and gas in a tidal stream between two interacting galaxies. The interaction between the two galaxies is believed to be the trigger of the starburst and through this exhaust the molecular gas supply in the AGN host galaxy. One scenario which could have triggered the high star formation in Freja, Thor, and Odin and perhaps the AGN activity in Yggdrasil is, like for 4C60.07, interaction between two galaxies. In this scenario, the two galaxies interact tidally where gas flows between the galaxies trigger the AGN activity in Yggdrasil, and creates tidal tails of gas which may emit Ly $\alpha$. The tidal interactions may then ignite the string of components Freja, Thor, and Odin resulting in a SFR $\sim 400 M_{\odot} / \mathrm{yr}$. However, the $\mathrm{CO}(8-7)$ emission line from Yggdrasil is very narrow and dynamically quiescent. It shows no signs of dynamical motion, implying that this gas is not related to the tidal interaction. The $\mathrm{CO}$ gas appears to be quite highly excited, as may be expected for gas near the central AGN. However, we note that the ATCA upper limit on the $\mathrm{CO}(1-0)$ emission is rather shallow, so we cannot put strong constraints on the $\mathrm{CO}$ gas excitation.

Along the same projected line as the bridge and dust emitting regions, we find $\mathrm{CO}(8-7)$ emission in Loke which is not detected in any optical or dust continuum counterpart. The tentative detection of $\mathrm{CO}(1-0)$ at the position of Loke is consistent with the presence of a large reservoir of molecular gas. However, it is not clear from the observations at hand how extended this reservoir is, if it reaches across Freja, Thor, Odin, and Loke, tracing one compact region, or if the reservoir is only at the location of Loke. The tentative $\mathrm{CO}(1-0)$ detection suggests a lower excitation level than Yggdrasil, but more sensitive low$J$ observations are needed to confirm this. The only thing known is that this region contains dynamically quiet but likely highly excited gas component $\sim 90 \mathrm{kpc}$ away from the nearest stellar emission and with a relatively low velocity compared to the systemic velocity of the AGN. A clue to its nature lies in the alignment with the AGN and starburst components along the bridge. If this represents an accretion flow of galaxies and material along this direction, the gas would experience tidal forces and accretion shocks when the galaxies get close (or enter the halo of Yggdrasil). The absorption component 2 reveals the presence of 
Table 6. Stellar mass, ionised-, neutral- and molecular gas mass for Yggdrasil and Thor.

\begin{tabular}{lcc}
\hline \hline Mass & $\begin{array}{c}\text { Yggdrasil } \\
M_{\odot}\end{array}$ & $\begin{array}{c}\text { Freja/Thor/Odin/Loke } \\
M_{\odot}\end{array}$ \\
\hline$M_{\text {stellar }}$ & $1.7 \times 10^{11}$ & $1 \times 10^{11}$ \\
$M_{\text {ion }}$ & $5.2 \times 10^{8}$ & - \\
$M_{\text {neutral }}$ & $3.8 \times 10^{9}$ & - \\
$M_{\text {mol }}$ & $6 \times 10^{10}$ & $2.3 \times 10^{10}$ \\
\hline
\end{tabular}

a large amount of neutral gas at a radius $>60 \mathrm{kpc}$ from the AGN, however the impact on this gas from merging galaxies or accreting gas is unknown. It may be that as gas enters the halo, it will encounter the large scale neutral gas, perhaps shocking it as it penetrates. Only more sensitive studies of the molecular gas in Loke may this impact be revealed.

\subsection{Distribution of masses}

To understand the nature of the MRC0943-242 system, it is important to know how the mass is distributed in the system. Following De Breuck et al. (2003), we estimate the ionised gas masses of Yggdrasil. The mass of the ionised gas is given by $M_{\text {ion }}=10^{9}\left(f_{-5} L_{44} V_{70}\right)^{1 / 2} M_{\odot}$, where $f_{-5}$ is the filling factor in units of $10^{-5}, L_{44}$ is the Ly $\alpha$ luminosity in units of $10^{44} \mathrm{erg} / \mathrm{s}$ and $V_{70}$ is the volume in units of $10^{70} \mathrm{~cm}^{3}$. We assume a filling factor of $10^{-5}$ (McCarthy et al. 1990) and a volume of $5^{\prime \prime} \times 2.5^{\prime \prime} \times 2.5^{\prime \prime}$, and therewith find $M_{\text {ion }}=5.2 \times 10^{8} M_{\odot}$. This is a lower limit to the total mass since the absorption in the Ly $\alpha$ emission is significant. Since the distribution of the absorption components 1 , 3 , and 4 are only delineated by the Ly $\alpha$ emission, it is unknown how this gas is distributed, meaning we are most likely underestimating their column densities as the components do not fully cover the emission. This means we cannot estimate their contributions to the mass budget.

Absorption component 2 is observed in all the Ly $\alpha$ line profiles across the Ly $\alpha$ emitting regions and must therefore cover the entire Ly $\alpha$ emitting region out to at least $\sim 60 \mathrm{kpc}$. Assuming it is distributed as a spherical shell, we estimate the total mass of absorption component 2 again following De Breuck et al. (2003), $M_{\text {neutral }} \gtrsim 3.8 \times 10^{9}(R / 60 \mathrm{kpc})^{2}\left(N_{\mathrm{HI}} / 10^{19}\right) \mathrm{cm}^{-2} M_{\odot}$, where $R$ is the radius of the neutral gas and $N_{\mathrm{HI}}$ is the HI column density. We note that because the absorption is heavily damped (Jarvis et al. 2003), our column density estimate is likely a lower limit of the true column, and our mass estimate therefore a lower limit.

Using the tentative $\mathrm{CO}(1-0)$ detection at the position of Loke, and choosing a conservative $\alpha_{\mathrm{CO}}=0.8 M_{\odot} \mathrm{K} \mathrm{km} \mathrm{s}^{-1} \mathrm{pc}^{-2}$ (Downes \& Solomon 1998) we estimate a molecular gas mass of $2.3 \times 10^{10} M_{\odot}$ for Loke. Emonts et al. (2011) discovered a tentative $3 \sigma \mathrm{CO}(1-0)$ detection $\sim 60 \mathrm{kpc}$ north-east of Yggdrasil, and estimate a molecular gas mass of $M_{\mathrm{H}_{2}}=6 \times 10^{10} M_{\odot}$, using the same value for $\alpha_{\mathrm{CO}}$.

It is clear from comparing the four mass estimates (Table 6) that the most of the mass is in the form of stars and molecular gas, although the contribution of the neutral shell of gas may also be significant.

\subsection{Ionisation mechanism}

The high ionisation lines such as HeII, CIV, and CIII] are extended beyond the western lobe and asymmetric on large scales (see panels A-C of Fig. 7). The simplest interpretation is a strong asymmetry in the gas distribution. This hypothesis is supported by the hard photons, which are most likely responsible for the ionisation of the gas, causing the asymmetry in the emission from the circum nuclear gas. Moreover, the ALMA data show a similar asymmetry in the distribution of both the dust and $\mathrm{CO}$ emission. However, this emitting gas lies outside of the ionisation cone and can therefore not be affected.

The detected emission lines from Ly $\alpha$, CIV, HeII, CIII], and CII] can be used as probes for the ionisation mechanisms. Both the AGN and the far-UV field of vigorous star-formation can ionise the gas. The line ratios of $\operatorname{Ly} \alpha$, CIV, and HeII can be used to determine the contribution from stellar photoionisation (Fig. 4 of Villar-Martín et al. 2007). We find that the observed (absorption corrected) line ratios of Yggdrasil (Table 1) are consistent with $\omega=0$, i.e. pure AGN photo-ionisation. Similarly, we can use the CIV/HeII, CIII]/HeII, CIV/CIII], CII]/CIII], CIV/Ly $\alpha$ and $\mathrm{CIII}] / \mathrm{Ly} \alpha$ flux ratios along with the diagrams of De Breuck et al. (2000; their Fig. 13, adapted in Fig. A.1). This comparison shows that the gas within the radio lobes of Yggdrasil, is likely ionised by a combination of photo-ionisation $(\sim 70 \%)$ and shock + precursors $(\sim 30 \%)$. CII] emission is an especially sensitive tracer of shock-ionised gas (Best et al. 2000; De Breuck et al. 2000). Our MUSE data allow us to produce a CII] narrow-band (moment-0) image (see panel D of Fig. 7). Interestingly, this image indeed reveals that the $\mathrm{CII}]$ emission is confined within the radio lobes, as would be expected if this line is dominated by shock ionised gas. Deeper MUSE data would be needed to check if this is not just due to limited $\mathrm{S} / \mathrm{N}$. This also limits us to derive line ratios in the extended halo (near Bifrost), where we detect Ly $\alpha$, but no other emission lines. Deeper data would allow us to check whether the gas at $\sim 80 \mathrm{kpc}$ is still ionised by the AGN, or has a more important contribution from stellar photo-ionisation or shocks.

\subsection{AGN and starbursts}

Disentangling the SED into several components suggests that the starburst activity in $\mathrm{Hz}$ RGs is driven by the interaction of two (or more) gas-rich systems. Major mergers have been invoked to explain the high SFRs seen in submm galaxies (SMGs; e.g. Engel et al. 2010), though others argue that they may rather represent the top end of a "main sequence" of star-forming galaxies (e.g. Michałowski et al. 2012). The similar morphologies and stellar masses of $\mathrm{Hz}$ RGs and SMGs suggest they may be related, potentially through an evolutionary sequence, a high- $z$ extension of the local relation between QSOs and ULIRGs (Sanders et al. 1988). Both classes reside in parent halos of similar mass (Hickox et al. 2012), but direct observations of objects in transition between SMGs and QSOs remain restricted to a few examples (e.g. Simpson et al. 2012). This is where detailed observational studies of type 2 AGN like HzRGs can play an important role, as their stellar masses, unlike those type 1 AGN, can be accurately determined (Seymour et al. 2007; De Breuck et al. 2010).

In MRC0943-242, we actually observe both processes: in Yggdrasil, we observe an AGN host galaxy with a moderate SFR, while the companion galaxy Thor is surrounded by two additional star-forming companions, Odin and Freja.

\subsection{Loke is not so atypical around radio galaxies}

Our detection of $\mathrm{CO}(8-7)$ emission in Loke, which is not coincident with any dust or stellar emission, and is not in the 
B. Gullberg et al.: The mysterious morphology of MRC0943-242 as revealed by ALMA and MUSE

Table 7. Overview of CO detections that are not directly associated with the radio galaxy.

\begin{tabular}{llcccc}
\hline \hline HzRG & $z$ & CO detection & Separation $^{a}$ & $\mathrm{CO} /$ dust association & Reference \\
\hline MRC0114-211 & 1.402 & $\mathrm{CO}(1-0)$ & $35 \mathrm{kpc}$ & no & Emonts et al. (2014); Nilsson et al. (in prep.) \\
MRC0152-209 & 1.921 & $\mathrm{CO}(1-0) / \mathrm{CO}(6-5)$ & $10 \mathrm{kpc}$ & yes & Emonts et al. (2014, 2015) \\
MRC0156-252 & 2.016 & $\mathrm{CO}(1-0)$ & $60 \mathrm{kpc}$ & no & Emonts et al. (2014); Nilsson et al. (in prep.) \\
MRC2048-272 & 2.060 & $\mathrm{CO}(1-0)$ & $55 \mathrm{kpc}$ & no & Emonts et al. (2014); Nilsson et al. (in prep.) \\
MRC1138-262 & 2.161 & $\mathrm{CO}(1-0)$ & $30-40 \mathrm{kpc}$ & yes & Emonts et al. (2013); Gullberg et al. (in prep.) \\
TXS0828+193 & 2.572 & $\mathrm{CO}(3-2)$ & $80 \mathrm{kpc}$ & no & Nesvadba et al. (2009) \\
MRC0943-242 & 2.923 & $\mathrm{CO}(8-7)$ & $80 \mathrm{kpc}$ & no & This work \\
B3J2330+3927 & 3.086 & $\mathrm{CO}(1-0) / \mathrm{CO}(4-3)$ & $30 \mathrm{kpc}$ & yes & Ivison et al. (2012) \\
4C41.17 & 3.798 & $\mathrm{CO}(4-3)$ & $13 \mathrm{kpc}$ & yes & De Breuck et al. (2005) \\
4C60.07 & 3.8 & $\mathrm{CO}(1-0) / \mathrm{CO}(4-3)$ & $30 \mathrm{kpc}$ & yes & Ivison et al. (2008), Greve et al. (2004) \\
\hline
\end{tabular}

Notes. ${ }^{(a)}$ Separation between the AGN and the CO detection.

direction of the axis of the radio emission, is puzzling. It is not the first time $\mathrm{CO}$ line emission has been detected around $\mathrm{a} \mathrm{H}_{z} \mathrm{RG}$ without any counterpart at other wavelengths. Table 7 lists ten $\mathrm{H} z \mathrm{RG}$ with $\mathrm{CO}$ observations where the $\mathrm{CO}$ emission is offset from the AGN compared to the radio sources.

Nesvadba et al. (2009) found $\mathrm{CO}(3-2)$ emission $80 \mathrm{kpc}$ from the core of the $z=2.6 \mathrm{HzRG}$ TXS0828+193. However, the $\mathrm{CO}(3-2)$ emission in TXS0828+193 is aligned with the radio jet, which Nesvadba et al. (2009) suggest could be triggering the collapse and excitation of the gas. The offset of CO emission from the position of the $\mathrm{HzRG}$ is seen in other sources as well (see Table 7) such as, 4C60.07 (Ivison et al. 2008), MRC0114211, MRC0156-252 and MRC2048-272 (Emonts et al. 2014). Ivison et al. (2008) detect $\mathrm{CO}(4-3)$ emission for the $\mathrm{HzRG}$ at $z=3.8$ at two positions: at the location of the AGN core and $7 " \mathrm{SW}$ of the AGN. The latter component is also detected in $\mathrm{CO}(1-0)$ emission (Greve et al. 2004). Based on the submm observations of 4C60.07, Ivison et al. (2008) point out a complication when calculating dynamical masses using the extended $\mathrm{CO}$ emission, due to the misalignment of the black hole with the $\mathrm{CO}$ emission and dust continuum. Emonts et al. (2014) discovered $\mathrm{CO}(1-0)$ emission in three $\mathrm{H}_{z} \mathrm{RG}$ s that were significantly offset from the AGN and which have 4.5-9.2 $\times 10^{10} M_{\odot}$ of molecular gas, but again aligned with the radio jet. They discuss jet-induced star formation. Two molecular components traced by $\mathrm{CO}(4-3)$ for 4C41.17 were detected by De Breuck et al. (2005). These two components are also aligned with the radio jet, but are co-spatial with the $\operatorname{Ly} \alpha$ emission.

The submm and optical observations of MRC 0943-242, adds to this complicated situation, with its many components, only two of which are traced by $\mathrm{CO}$ emission.

\section{Are we seeing a multiphase accretion flow?}

Numerical simulations suggest that galaxies acquire much of their gas through accretion flows generated by the growth of the cosmic web of dark matter (e.g. Agertz et al. 2011; Danovich et al. 2015). Flows of gas develop over cosmological distances and time scales, ultimately penetrating into halos and feeding baryons onto galaxies. Because the flows are associated with the growth of dark matter structures, galaxies are expected to be embedded in accretion flows. Could we be witnessing such accretion in our combined data sets?

Progressing inwards in radius towards the host galaxy of MRC 0943-242 over many 10s of kpc from Loke, through Odin, Thor, Freja, Bifrost to the redshifted absorption component 3 seen against the inner Ly $\alpha$ emission, we see little change in the velocity over this inward journey. In the most distant emission, i.e. Loke, the velocity offset relative to the AGN emission is small. This structure appears dynamically cold, having low dispersion in all of its spectroscopic features, implying that it is near to the plane of the sky. Aligned with this general structure is a group of (merging?) vigorous star-forming galaxies associated with the emission regions, Freja, Thor, and Odin. If the coincident Ly $\alpha$ emission from Bifrost is representative of their projected velocity, these galaxies are embedded within this overall (in projection) linear structure. On the opposite end of the structure, we find the AGN host galaxy, Yggdrasil, which has a rather mundane rest-frame optical morphology, suggesting a heavily obscured galaxy, but not a merger (see Fig. 9 of Pentericci et al. 2001). Interestingly, the extended emission line halo does not extend on the other side of the AGN.

Admittedly, we do not have a simple explanation for the distribution of the various phases. For example, why is most of the molecular gas observed at the position of Loke? An explanation could be that the molecular gas is related to the strong, optically thick, large covering fraction of HI absorption component 2. However, the velocity offsets (Fig. 4) suggest that it is rather the less massive absorption component 3 that coincides with the $\mathrm{CO}(8-7)$ and simply part of what may be a flow into Yggdrasil.

Only a combined ALMA + MUSE survey of radio galaxies like that of MRC0943-242 (with deeper integrations than in this pilot project) can determine if it is gas accretion that is fuelling the growth of their galaxies and supermassive black holes. These combined data cubes are our only way to obtain a complete picture of various phases which emit strongly over a wide range of wavelengths. This will allow us to study the nature of the halo gas that results from this complex interaction. The sensitivity of both ALMA and MUSE on the VLT are already offering tantalising clues on how the gas in this system is being acquired and modified by the interaction with the AGN and galaxies in this complex system.

\section{Conclusions}

Combining the high sensitivity of ALMA and MUSE, even with very modest integration times, has allowed us to gain insights into the environments that explain many properties of the evolutionary path of $\mathrm{H}_{z} \mathrm{RGs}$. Surprisingly, in our pilot study of the $z=2.9$ radio galaxy MRC0943-242, we apparently find that most of the star formation is not associated with the radio galaxy itself, but is in a companion set of galaxies that exhibit a complex pattern of dust emission which we associate with star formation. 
We say apparently because the morphology of the dust emission is peculiarly distributed in three distinct sources but roughly within the diffuse stellar continuum emission of the source.

The MUSE data show that the continuum emitting source is embedded in a Ly $\alpha$ emitting linear filament stretching from beyond the AGN to the circum nuclear emission of the AGN. This structure of dust and Ly $\alpha$ emission aligns well in projection with a region of $\mathrm{CO}(8-7)$ and $\mathrm{CO}(1-0)$ emitting gas (at lower significance) about $90 \mathrm{kpc}$ in projection from the AGN.

The AGN apparently ionises a large region which is asymmetric, lying mostly on the western side of the source. This is likely due to the gas distribution as it tantalisingly lies in the direction where the dust, $\mathrm{CO}$ line emission, and the filamentary Ly $\alpha$ emission are found.

The relationship between the Ly $\alpha$ emission and absorption and the associate CIV absorption paints a fascinating picture of the distribution of the warm ionised medium. There is a component of $\operatorname{Ly} \alpha$ absorbing gas which reaches zero intensity throughout the Ly $\alpha$ emission detected across MRC0943-242 in the MUSE data. It clearly has a unity covering fraction and is highly optically thick. Since the gas is seen as an absorption feature throughout the Ly $\alpha$ emitting filament (Bifrost) this thick, unity covering fraction absorber is extended and is visible in absorption out to $>60 \mathrm{kpc}$. Because of its velocity and optical thickness, it absorbs all of the extended Ly $\alpha$ emission in the cube.

Acknowledgements. We thank the anonymous referee for her/his very thorough reading of our manuscript, and suggestions that substantially improved our paper. This publication uses data taken from the MUSE commissioning run 060.A-9100. All of the MUSE data used in this paper are available through the ESO science archive. This paper makes use of the following ALMA data: ADS/JAO.ALMA\#2012.1.00039.S. ALMA is a partnership of ESO (representing its member states), NSF (USA) and NINS (Japan), together with NRC (Canada) and NSC and ASIAA (Taiwan), in cooperation with the Republic of Chile. The Joint ALMA Observatory is operated by ESO, AUI/NRAO and NAOJ. The work of D.S. was carried out at Jet Propulsion Laboratory, California Institute of Technology, under a contract with NASA. B.E. acknowledges funding through the European Union FP7 IEF grant nr. 624351. M.D.L. acknowledges the support from the ESO visitors program and especially would like to thank Mario van den Ancker for his help and Eric Emsellem for interesting scientific discussions. Nick Seymour is the recipient of an ARC Future Fellowship. We thank Kurt Soto for giving us access to the ZAP software which suppresses residuals of sky subtraction using a principal component analysis.

\section{References}

Agertz, O., Teyssier, R., \& Moore, B. 2011, MNRAS, 410, 1391 Archibald, E. N., Dunlop, J. S., Hughes, D. H., et al. 2001, MNRAS, 323, 417 Bacon, R., Accardo, M., Adjali, L., et al. 2010, in SPIE Conf. Ser., 7735, 8 Bacon, R., Vernet, J., Borisova, E., et al. 2014, The Messenger, 157, 13 Bekki, K. 1998, ApJ, 504, 50

Best, P. N., Röttgering, H. J. A., \& Longair, M. S. 2000, MNRAS, 311, 23
Binette, L., Kurk, J. D., Villar-Martín, M., \& Röttgering, H. J. A. 2000, A\&A, 356,23

Carilli, C. L., Röttgering, H. J. A., van Ojik, R., et al. 1997, ApJS, 109, 1

Danovich, M., Dekel, A., Hahn, O., Ceverino, D., \& Primack, J. 2015, MNRAS, 449, 2087

De Breuck, C., Röttgering, H., Miley, G., van Breugel, W., \& Best, P. 2000, A\&A, 362, 519

De Breuck, C., van Breugel, W., Stanford, S. A., et al. 2002, AJ, 123, 637

De Breuck, C., Neri, R., Morganti, R., et al. 2003, A\&A, 401, 911

De Breuck, C., Downes, D., Neri, R., et al. 2005, A\&A, 430, L1

De Breuck, C., Seymour, N., Stern, D., et al. 2010, ApJ, 725, 36

Downes, D., \& Solomon, P. M. 1998, ApJ, 507, 615

Drouart, G., De Breuck, C., Vernet, J., et al. 2014, A\&A, 566, A53

Eales, S., Rawlings, S., Law-Green, D., Cotter, G., \& Lacy, M. 1997, MNRAS, 291, 593

Emonts, B. H. C., Norris, R. P., Feain, I., et al. 2011, MNRAS, 415, 655

Emonts, B. H. C., Feain, I., Röttgering, H. J. A., et al. 2013, MNRAS, 430, 3465

Emonts, B. H. C., Norris, R. P., Feain, I., et al. 2014, MNRAS, 438, 2898

Emonts, B. H. C., De Breuck, C., Lehnert, M. D., et al. 2015, A\&A, 584, A99

Engel, H., Tacconi, L. J., Davies, R. I., et al. 2010, ApJ, 724, 233

Greve, T. R., Ivison, R. J., \& Papadopoulos, P. P. 2004, A\&A, 419, 99

Hickox, R. C., Wardlow, J. L., Smail, I., et al. 2012, MNRAS, 421, 284

Ivison, R. J., Morrison, G. E., Biggs, A. D., et al. 2008, MNRAS, 390, 1117

Ivison, R. J., Smail, I., Amblard, A., et al. 2012, MNRAS, 425, 1320

Jarvis, M. J., Wilman, R. J., Röttgering, H. J. A., \& Binette, L. 2003, MNRAS, 338,263

Kennicutt, Jr., R. C. 1998, ApJ, 498, 541

Laurent, G. T., Aguirre, J. E., Glenn, J., et al. 2005, ApJ, 623, 742

Lilly, S. J., \& Longair, M. S. 1984, MNRAS, 211, 833

McCarthy, P. J., Spinrad, H., Dickinson, M., et al. 1990, ApJ, 365, 487

Michałowski, M. J., Dunlop, J. S., Cirasuolo, M., et al. 2012, A\&A, 541, A85

Mullaney, J. R., Alexander, D. M., Goulding, A. D., \& Hickox, R. C. 2011, MNRAS, 414, 1082

Nesvadba, N. P. H., Neri, R., De Breuck, C., et al. 2009, MNRAS, 395, L16

Ogle, P., Whysong, D., \& Antonucci, R. 2006, ApJ, 647, 161

Pentericci, L., McCarthy, P. J., Röttgering, H. J. A., et al. 2001, ApJS, 135, 63

Reuland, M., van Breugel, W., Röttgering, H., et al. 2003, ApJ, 592, 755

Reuland, M., Röttgering, H., van Breugel, W., \& De Breuck, C. 2004, MNRAS, 353,377

Röttgering, H. J. A., Hunstead, R. W., Miley, G. K., van Ojik, R., \& Wieringa, M. H. 1995, MNRAS, 277, 389

Sanders, D. B., Soifer, B. T., Elias, J. H., et al. 1988, ApJ, 325, 74

Seymour, N., Stern, D., De Breuck, C., et al. 2007, ApJS, 171, 353

Seymour, N., Altieri, B., De Breuck, C., et al. 2012, ApJ, 755, 146

Simpson, C., Rawlings, S., Ivison, R., et al. 2012, MNRAS, 421, 3060

Swinbank, A. M., Vernet, J. D. R., Smail, I., et al. 2015, MNRAS, 449, 1298

Tremaine, S., Gebhardt, K., Bender, R., et al. 2002, ApJ, 574, 740

van Breugel, W. J. M., Stanford, S. A., Spinrad, H., Stern, D., \& Graham, J. R. 1998, ApJ, 502, 614

van Ojik, R., Roettgering, H. J. A., Carilli, C. L., et al. 1996, A\&A, 313, 25

Venemans, B. P., Röttgering, H. J. A., Miley, G. K., et al. 2007, A\&A, 461, 823

Vernet, J., Fosbury, R. A. E., Villar-Martín, M., et al. 2001, A\&A, 366, 7

Villar-Martín, M., Vernet, J., di Serego Alighieri, S., et al. 2003, New Astron. Rev., 47, 291

Villar-Martín, M., Humphrey, A., De Breuck, C., et al. 2007, MNRAS, 375, 1299

Weilbacher, P. M., Streicher, O., Urrutia, T., et al. 2012, in SPIE Conf. Ser., 8451, 84510B

Weiß, A., Downes, D., Neri, R., et al. 2007, A\&A, 467, 955

Wylezalek, D., Galametz, A., Stern, D., et al. 2013, ApJ, 769, 79 
B. Gullberg et al.: The mysterious morphology of MRC0943-242 as revealed by ALMA and MUSE

\section{Appendix A: Line ratio diagrams from De Breuck et al. (2000)}

This appendix presents a range of emission line ratio diagrams that are directly derived from the MUSE data, compared photo-ionisation and shock ionisation models (Fig. 13 from De Breuck et al. 2000).
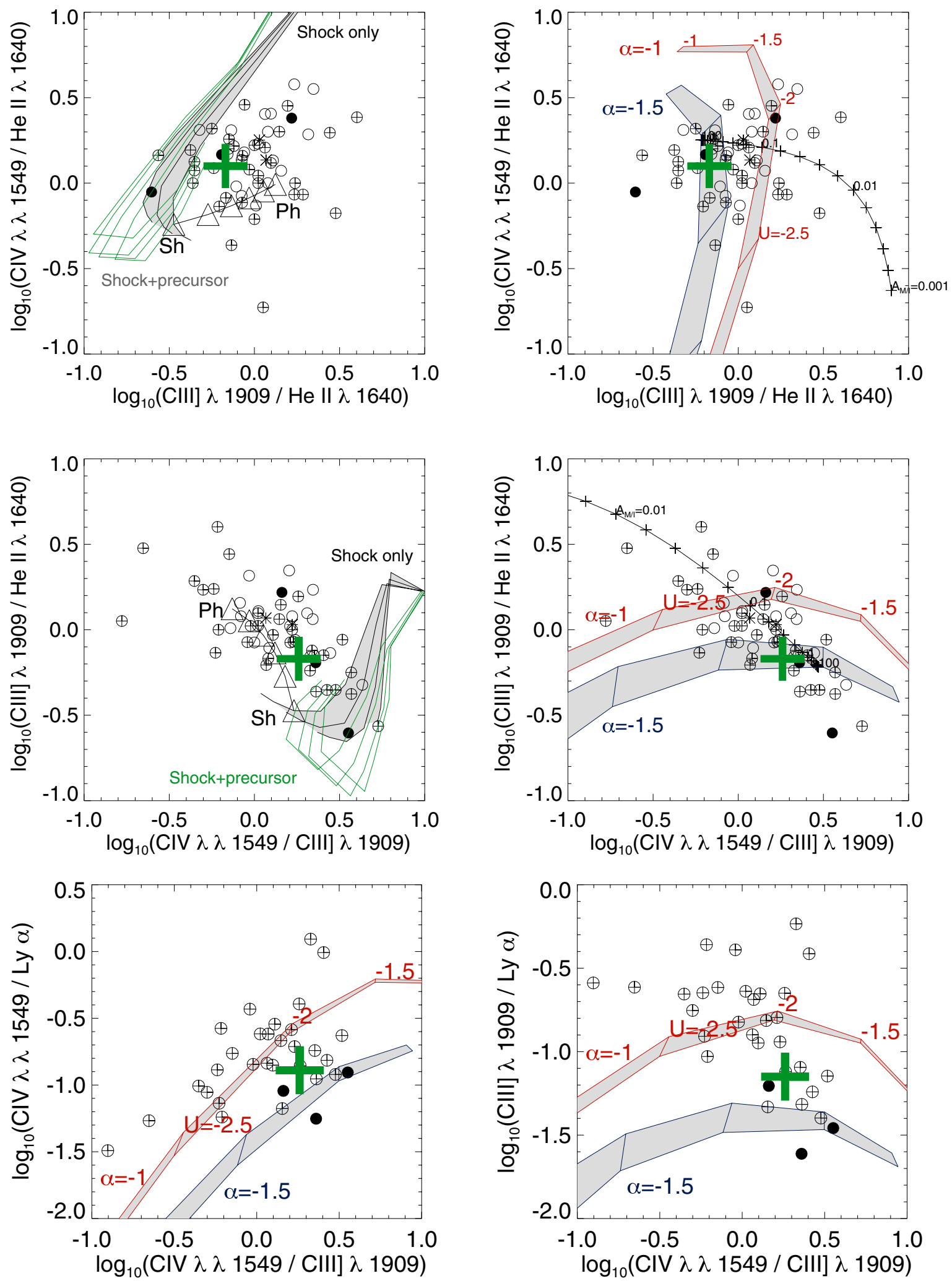

Fig. A.1. Line ratio diagnostic diagrams involving Ly $\alpha$, CIV, HeII, CIII], and CII] (adapted from De Breuck et al. 2000, their Fig. 13). The thick green cross shows the flux line ratios observed for Yggdrasil, while the circles show other HzRGs from the literature. Also shown are photoionisation and shock models to illustrate that the observed line ratios in Yggdrasil are dominated by photo-ionisation with a contribution of up to $\sim 30 \%$ by shocks (see De Breuck et al. 2000, for more details). 